\title{
Spectra and magnetic properties of large spins in external fields
}

\author{
V. A. Kalatsky ${ }^{1}$ and V. L. Pokrovsky ${ }^{1,2}$ \\ ${ }^{1}$ Department of Physics, Texas A \& M University, College Station, Texas 77843-4242 \\ ${ }^{2}$ Landau Institute for Theoretical Physics, Kosygin Street 2, Moscow 117940, Russia
}

(Received 24 December 1998)

\begin{abstract}
Spectra and magnetic properties of large spins $J$ (e.g., spins possessed by ions or molecules), placed into a crystal electric field (CEF) of an arbitrary symmetry point group, are shown to change drastically when $J$ changes by $1 / 2$ or 1 . At a fixed field symmetry and configuration of its $N$ extrema situated at the $p$-fold symmetry axis, physical characteristics of the spin depend periodically on $J$ with the period equal to $p$. The problem of the spectrum and eigenstates of the large spin $J$ is equivalent to an analogous problem for a scalar charged particle confined to a sphere $S^{2}$ and placed into the magnetic field of the monopole with the charge $J$. This analogy, as well as the strong difference between close values of $J$, stems from the Berry phase occurring in the problem. For energies close to the extrema of the CEF, the problem can be formulated as Harper's equation on the sphere. The $(2 J+1)$-dimensional space of states is split into smaller multiplets of classically degenerated states. These multiplets in turn are split into submultiplets of states transforming according to specific irreducible representations of the symmetry group determined by $J$ and $p$. We classify possible configurations and corresponding spectra. Experimental realizations of large spins in a symmetric environment are proposed and physical effects observable in these systems are analyzed. [S1050-2947(99)00709-X]

PACS number(s): 03.65.Sq, 03.65.Bz, 75.10.Dg, 02.20.Df
\end{abstract}

\section{INTRODUCTION}

Conventional wisdom accepts that large spins or orbital momenta $J$ (in units of $\hbar$ ) are almost classical. In particular, if $J \gg 1$, their measurable properties do not change substantially if $J$ changes by $1 / 2$ or 1 . This common belief was undermined by Haldane [1], who demonstrated that the ground state and spectrum of the low-energy states in onedimensional spin chains are absolutely different for integer and half-integer spins.

In this paper we show that similar phenomena can be observed on the level of an individual spin placed into external electric field. If the field possesses high symmetry (cubic or icosahedral) the distinction between spins becomes more subtle. For example, in the case of cubic symmetry not only do integer spins differ from half integer (this difference is intuitively obvious due to the Kramers degeneracy), but the remainder at division of the spin by 4 determines the spectrum and degeneracy of the low-lying states. These striking differences can be found in experiment either by spectral analysis or by magnetic measurements. We will show that spins 1000, 1001, and 1002 placed into a cubic environment have $100 \%$ different magnetic susceptibilities at low temperature. Moreover, we will show that a kind of randomness appears in properties of large spins in some cases and variation of large spins by one can change magnetic and spectral properties in an uncontrollable way.

Certainly, the conventional wisdom we started with is presumably correct. It is wrong only in a very small range of energy or temperature, the smaller the larger is $J$. Nevertheless, as already happened with the Haldane theory, these deviations from classical behavior may be important for the experiment.

The source of all these peculiarities is Berry's phase. Physically, it is associated with the fact that, when the classical rotator moves on its unit sphere, it simultaneously ro- tates around its axis. The rotation phase distinguishes the rotator from a quantum or classical particle confined on a sphere. The rotator problem can be reduced to the particle problem, but the representing particle must have an electric charge of unity and must be subjected to the homogeneous magnetic field of a monopole with the magnetic charge $J$ placed into the center of the sphere. In quantum mechanics $J$ accepts integer and half-integer values. Since the phases are determined modulo $2 \pi$, a change of $J$ by $1 / 2$ or 1 can substantially change phase factors, even if $J$ is large. Therefore, the tunneling amplitudes for close large $J$ can differ significantly by their phase, leading to different spectra.

Harter and Patterson [2] considered a similar problem in the context of the rotation-vibrational spectrum of cubically symmetric molecule $\mathrm{SF}_{6}$ [3]. Since Berry's phase was not invented at that time, their approach was ingenious but not physically transparent. They have invented a clever trick associated with the Frobenius duality theorem to avoid explicit introduction of Berry's phases, as we understand it today. Though the lack of clarity did not allow them to solve the problem completely, it is really surprising how far they were able to penetrate into the problem.

This paper is composed as follows. In the next section we introduce a quasiclassical description of large spins. Berry's phase, Berry's connection, and reduction to the problem of a charged particle in the monopole field are considered in Sec. III. In the fourth section we perform the group analysis of the problem. The fifth section contains the derivation of the lowenergy spectrum and magnetic properties of large spins. We separated the case of random levels in Sec. VI. Numerical calculations for a special potential in a wide range of spin values are given in Sec. VII. In Sec. VIII we propose experimental realizations of large spins. Our conclusions can be found in Sec. IX.

Brief reports on a part of this work were published earlier $[7,15]$. 


\section{QUASICLASSICAL DESCRIPTION OF LARGE SPINS}

The classical image of a large spin is the classical rotator, i.e., a vector with a fixed length $J$. Its position is determined by two spherical coordinates $\theta$ and $\phi$. Sometimes coordinates $J_{z}=J \cos \theta$ and $\phi$ are more convenient since they have simple Poisson brackets: $\left\{J_{z}, \phi\right\}=1$. Classical motion is determined by the Hamiltonian

$$
\mathcal{H}=f(\mathbf{J})-\mathbf{h} \cdot \mathbf{J},
$$

where $\mathbf{h}$ is magnetic field (with a precision of a constant factor) and $f(\mathbf{J})$ is an arbitrary function of $\mathbf{J}$, invariant with respect to inversion: $\mathbf{J} \rightarrow-\mathbf{J}$. The latter requirement is equivalent to the time-reversal symmetry [4]. Together with the standard Poisson brackets $\left\{J_{i}, J_{j}\right\}=\varepsilon_{i j k} J_{k}$ the Hamiltonian (1) contains full information on classical spin dynamics. Periodical trajectories on the sphere can be quantized according to the Bohr quantization rule:

$$
\oint J_{z}(\phi, E) d \phi=\left(n+\gamma_{B}\right) \pi,
$$

where $J_{z}(\phi, E)$ can be found from the equation $f(\mathbf{J})=E$ with the substitution $J_{x}=\sqrt{J^{2}-J_{z}^{2}} \cos \phi, J_{y}=\sqrt{J^{2}-J_{z}^{2}} \sin \phi$, and $\gamma_{B}$ is a constant.

Let us first consider general properties of spin trajectories in zero magnetic field. The function $f(\mathbf{J})$, being continuous on the sphere, has at least two minima and two maxima. If the external crystal field has a nontrivial symmetry group, the number of equivalent minima is larger. For example, it can be equal to 4 for tetragonal symmetry, 6 for hexagonal symmetry. In the case of cubic symmetry it can be 6,8 , or 12 (directed along fourfold, threefold, and twofold axes, respectively). The number of equivalent minima for icosahedral symmetry can be 12, 20, and 30 (directed along fivefold, threefold, and twofold axes, respectively). We considered the situations when extrema are located in the symmetrical positions. In principle, it is possible that they are in more general asymmetric positions.

Classical trajectories can be separated into two classes: "localized" and "delocalized." If energy is close enough to the minimum (maximum) of $f(\mathbf{J})$, the trajectories are confined in the vicinity of one of the minima (maxima). We call such trajectories localized. In the intermediate region of the energy trajectories are "delocalized," they are not confined near any of the extrema. It is obvious that delocalized trajectories are highly model dependent, i.e., they depend on a specific form of $f(\mathbf{J})$. Localized trajectories are much more universal: they depend only on the symmetry and on the positions of the minima. The same remark is correct with respect to quantized levels: low-lying levels, close to $f_{\min }$, or almost maximal values of energy, close to $f_{\max }$, have universal features, whereas levels in between are rather nonuniversal. Therefore, we will study only a part of the spectra close to $f_{\min }$ or $f_{\max }$. Note that the spectrum of the quantum problem is discrete and limited by $f_{\min }$ and $f_{\max }$.

Before we proceed to detailed study of these levels let us make an important remark. For any fixed $J$ and any given $f(\mathbf{J})$ the quantum problem consists in the diagonalization of the $(2 J+1) \times(2 J+1)$ matrix. Therefore, the question arises whether the general theory is necessary. The answer is yes,

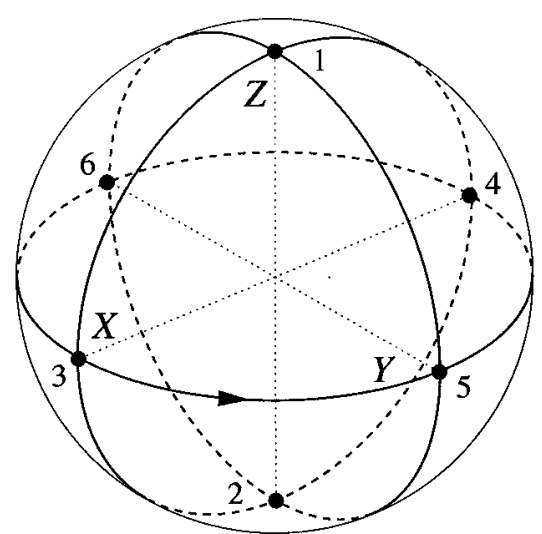

FIG. 1. Tunneling trajectories of the spin (single paths). The sixfold configurations of $\mathbf{O}$.

because no reliable information about function $f(\mathbf{J})$ is available. We present here general facts, independent of the specific form of $f(\mathbf{J})$, but only on its symmetry group and specific configuration of the extrema. The only requirement for our theory is $J \gg 1$.

Thus, classically a localized stationary state is multiply $(N$-fold) degenerate. Quantum fluctuations provide a finite radius for each of these states which can be enumerated as $|1\rangle,|2\rangle, \ldots,|N\rangle$. For the large $J$ considered all these states are oscillatory ones within the precision $1 / J$. A more subtle, but not least essential, quantum effect is the tunneling between these states. The tunneling amplitude between two states $|i\rangle$ and $|j\rangle, \quad i \neq j$, is exponentially small, $w_{i j}$ $\propto \exp \left(-c_{i j} J\right)$, where $c_{i j}$ are constants for a given $f(\mathbf{J})$. Therefore, we take into account only tunneling between the nearest-neighbor states, i.e., the ones with the smallest $c_{i j}$ $=c$, and neglect tunneling between more remote states with $c_{i j}>c$. To estimate the value of $c$, we need to specify the Hamiltonian. For simplicity we consider the case of the cubic symmetry with the Hamiltonian

$$
\mathcal{H}_{1}^{\mathbf{O}}=-a\left(J_{x}^{4}+J_{y}^{4}+J_{z}^{4}\right),
$$

where $a>0$ is a constant. The minimum value of $\mathcal{H}_{1}^{\mathbf{O}}$ is $E_{\min }=-a J^{4}$. There are six minima corresponding to the directions of the fourfold axes: $( \pm J, 0,0),(0, \pm J, 0),(0,0$, $\pm J)$. Let us consider, for example, tunneling between minima $(J, 0,0),(0, J, 0)$. By symmetry the tunneling trajectory is the smaller arc of the big circle passing through these points (Fig. 1). Setting $\mathcal{H}_{1}^{\mathbf{O}}=E_{\text {min }}$ we find from Eq. (3)

$$
J_{z}(\phi)= \pm i J \sqrt{\frac{1-\cos 4 \phi}{7+\cos 4 \phi}} .
$$

The tunneling amplitude is proportional to the exponent;

$$
w \propto \exp \left(i \int_{0}^{\pi / 2} J_{z}(\phi) d \phi\right)=\exp [-(J \ln 3) / 2]=e^{-0.55 J} .
$$

For a more realistic Hamiltonian

$$
\mathcal{H}_{2}^{\mathbf{o}}=\mathcal{H}_{1}^{\mathbf{o}}-b\left(J_{x}^{6}+J_{y}^{6}+J_{z}^{6}+30 J_{x}^{2} J_{y}^{2} J_{z}^{2}\right),
$$




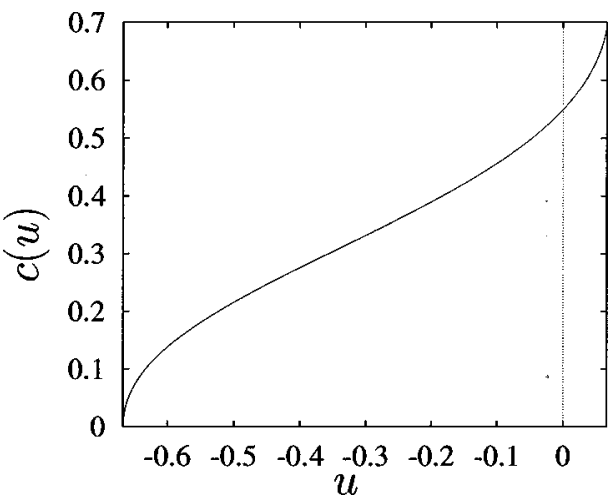

FIG. 2. $c(u)$ for the sixfold configuration of $\mathbf{O}$. Region of the single tunneling path regime.

the exponential factor in the tunneling amplitude is exp $[-c(u) J]$, where $c(u)$ is a function of the ratio $u=b J^{2} / a$. The graph of $c(u)$ is shown in Fig. 2 for values of $u$ in the interval $-2 / 3<u<1 / 15(a>0)$, where the tunneling path passes along the geodesics. In the region $1 / 15<u<3$ ( $a$ $>0$ ), the six minima are still global, however, the tunneling trajectories (there are two of them due to the symmetry) deviate from the geodesics (see Fig. 6) and the estimation of the exponent becomes more complicated. Effects of the multiple path tunneling, for the case of the octahedron configurations, will be considered in Sec. V. A numerical analysis of the Hamiltonian (6) and comparison to the predictions of the semiclassical approximation is given in Sec. VII.

Another important feature of the Hamiltonian (6) is that, depending on signs of $a$ and $b$ and the parameter $u$, it displays 6,8 , or 12 minima. The phase diagram for this, important for applications, Hamiltonian is shown in Fig. 3. On the boundaries of different "phases" different groups of minima become equal to each other. Then, in the quantum problem, the degeneracy of the ground state increases, for example, from 6 to 14. Therefore one can expect some singularities in the close vicinity of the boundaries.

For the case of the icosahedral symmetry the simplest Hamiltonian is

$$
\begin{aligned}
\mathcal{H}_{1}^{\mathbf{Y}}= & -a\left\{J_{x}^{6}+J_{y}^{6}+J_{z}^{6}+30 J_{x}^{2} J_{y}^{2} J_{z}^{2}-3 \sqrt{5}\left[J_{x}^{2} J_{y}^{2}\left(J_{x}^{2}-J_{y}^{2}\right)\right.\right. \\
& \left.\left.+J_{y}^{2} J_{z}^{2}\left(J_{y}^{2}-J_{z}^{2}\right)+J_{z}^{2} J_{x}^{2}\left(J_{z}^{2}-J_{x}^{2}\right)\right]\right\} .
\end{aligned}
$$

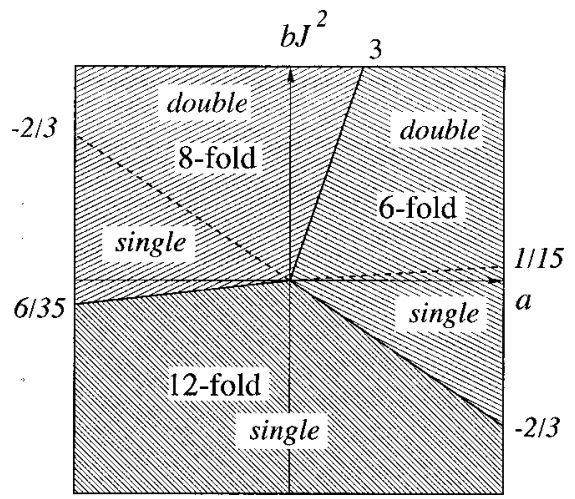

FIG. 3. Phase diagram of Hamiltonian (6) in $b J^{2}-a$ plane. The dashed lines separate the regions of the single and double path tunneling. The numbers on the periphery are the slopes of the corresponding lines.
The minimum value of $\mathcal{H}_{1}^{\mathbf{Y}}$ is $a J^{6} / 5 \quad(a<0)$. There are 12 minima corresponding to the vertices of an icosahedron (directions of the fivefold axes): $J(\alpha, \beta, 0), J(0, \alpha, \beta)$, $J(\beta, 0, \alpha)$, where $\alpha^{2}=(5+\sqrt{5}) / 10$ and $\beta^{2}=(5-\sqrt{5}) / 10$. A calculation similar to the one for $\mathcal{H}_{1}^{\mathbf{0}}$ gives the exponential part of the tunneling amplitude $\exp (-0.28 J)$. Positive values of $a$ yield the 20-fold configuration with the minima along the threefold axes.

Addition of the next nontrivial invariant of the icosahedron group, a polynomial of the tenth order over $\mathbf{J}$, allows the configuration with 30 minima along the twofold axes.

The tunneling partly lifts the classical degeneracy. What was the $N$-fold degenerate state without tunneling is split into a multiplet of sublevels separated by exponentially small energy intervals $\propto \exp (-c J)$, whereas the distances between different multiplets are proportional to $1 / J$. Each sublevel in the multiplet corresponds to a finite-dimensional subspace of states transforming according to an irreducible representation of the symmetry group. However, as we mentioned already, the realization of this group and the spectrum for the rotator are very different from those for a quantum particle confined on a sphere. Anyway the problem is reduced to diagonalization of a square matrix of the rank $N$ (classical degeneracy of the level) with nonzero matrix elements between geometrically closest states only. We neglect the tunneling between more remote states (non-nearest-neighbors) unless otherwise stated.

\section{BERRY'S PHASE, BERRY'S CONNECTION}

In the framework of quasiclassical spin dynamics the spin is treated as a rigid vector fixed by its direction $\mathbf{n}$. The closest quantum analog is the so-called coherent state $|\mathbf{n}\rangle$ which is defined as an eigenstate of operator $\mathbf{n} \cdot \mathbf{J}$ with the maximal eigenvalue $J$. Such a state has minimal uncertainty of the spin components transverse to the spin quantization axis [6]. An explicit construction for the coherent state reads [7]

$$
|\mathbf{n}\rangle=\exp \left(i J_{z} \phi\right) \exp \left(i J_{x} \theta\right) \exp \left(-i J_{z} \phi\right)|\hat{z}\rangle
$$

where $\theta$ and $\phi$ are spherical coordinates of $\mathbf{n} ;|\hat{\mathbf{z}}\rangle$ is the coherent state with the direction of quantization axis along the $z$ axis. This definition assures single valuedness of the spin wave function. An adiabatic motion of classical spin $\mathbf{n}(t)$ can be described by the coherent state $|\mathbf{n}(t)\rangle$ accompanied with a phase factor $e^{i \gamma}$ of purely geometrical origin [8]. Namely, if the spin moves adiabatically along any path $l$ on the unit sphere $S^{2}$ of $\mathbf{n}$, the geometrical phase $\gamma(l)$ is equal to a linear integral:

$$
\gamma=\int_{l} \mathbf{A}
$$

The local change of the phase is described by Berry's connection $A_{\mu}=\left\langle\mathbf{n}\left|i \partial / \partial x^{\mu}\right| \mathbf{n}\right\rangle$. This vector field has two components on $S^{2}$ :

$$
A_{\theta}=\left\langle\mathbf{n}\left|i \frac{\partial}{\partial \theta}\right| \mathbf{n}\right\rangle=0,
$$




$$
A_{\varphi}=\frac{1}{\sin \theta}\left\langle\mathbf{n}\left|i \frac{\partial}{\partial \varphi}\right| \mathbf{n}\right\rangle=J \frac{(1-\cos \theta)}{\sin \theta} .
$$

The connection $\mathbf{A}$, as well as the geometric phase, are not gauge invariant. At a local gauge transformation $|\mathbf{n}\rangle$ $\rightarrow \exp [i \lambda(\mathbf{n})]|\mathbf{n}\rangle$ they are transformed as follows: $\mathbf{A} \rightarrow \mathbf{A}$ $+\mathbf{d} \lambda, \gamma \rightarrow \gamma+\lambda_{f}-\lambda_{i}$, where $\lambda$ is an arbitrary differentiable function on $S^{2}$, and $i$ and $f$ are its values at the initial and final points of path $l$, respectively. However, the phase becomes gauge invariant if the path is closed: $l=\partial c$, where $c$ is a surface supported by $l$. In this case

$$
\gamma(c)=\int_{\partial c} \mathbf{A}=\int_{c} \mathbf{d} \mathbf{A}=J \int_{c} \sin (\theta) d \theta d \phi=J \Omega,
$$

where $\Omega$ is the solid angle subtended by $\partial c$ at the origin of the unit sphere. The integrand in Eq. (11) is the field strength $\mathbf{B}=J \hat{\mathbf{r}} / r^{2}$. This field is identical to magnetic field produced on $S^{2}$ by Dirac's magnetic monopole with the charge $J$ located in the center of the sphere. Thus, following Berry, we formulated the problem of a localized large spin in terms of a scalar charged particle confined on the sphere in the field of a magnetic monopole.

In the presence of a crystal field a further simplification becomes possible. As was shown in Sec. II, it leads to the localization of the low-energy states near the "easy" directions or minima of the field and lowers the dimensionality from $2 J+1$ to $N$, where $N$ is the number of the easy positions. The spin trapped near one of the easy directions can tunnel to the neighboring minima. The tunneling trajectories are solutions of the classical equations of motion with imaginary time or velocity. The amplitude $w_{i j}$ for the tunneling from the state $|i\rangle$ to a neighboring state $|j\rangle$ can be written as $w_{i j}=w \exp i \phi_{i j}$. Here $w$ is a real, exponentially small factor (see its calculation in Sec. II) and $\phi_{i j}$ is the Berry's phase along the tunneling trajectory connecting the points $i$ and $j$.

The set of Berry's phases $\phi_{i j}$ along the tunneling trajectories $\{i, j\}$ connecting extrema labeled by $i$ and $j$ must satisfy a set of equations. Namely, let us consider a plaquette $c$ on the sphere bounded by $k$ tunneling paths $\left\{i_{1}, i_{2}\right\},\left\{i_{2}, i_{3}\right\}, \ldots,\left\{i_{k}, i_{1}\right\}$. Then

$$
\sum_{m} \phi_{i_{m}, i_{m+1}}=\gamma(c)=J(\Omega(c)(\bmod 4 \pi)),
$$

where $i_{k+1}=i_{1}$ and $\Omega(c)$ is the solid angle subtended by the contour $\partial c$. The system (12) is extended over all independent plaquettes. Without loss of generality it is possible to consider Eq. (12) only for minimal (elementary) plaquettes, i.e., plaquettes of the minimal non-zero area whose boundaries do not have self-intersections. Equations (12) do not define the phases $\phi_{i j}$ unambiguously. There remains a freedom of a discrete gauge transformation $\phi_{i j} \rightarrow \phi_{i j}+f_{i}-f_{j}$ containing $N$ real parameters $f_{i}$. One of them can be treated as a common phase factor and is inessential. The Schrödinger equation in this representation reads

$$
\mathcal{H}|\psi\rangle=E|\psi\rangle,
$$

where $|\psi\rangle=\sum_{j=1}^{N} c_{j}|j\rangle$ is a vector in the $N$-dimensional space spanned onto the basis $|j\rangle, j=1,2, \ldots, N$ and $\mathcal{H}$ is an $N$ $\times N$ matrix whose diagonal components are equal to a singlewell energy level and nondiagonal elements are $\{\mathcal{H}\}_{i j}$ $=w_{i j}$. Further, we set the diagonal matrix elements of $\mathcal{H}$ to zero. Then Eq. (13) can be rewritten in the vector form

$$
\sum_{j=1}^{N} w_{i j} c_{j}=E c_{i}, \quad w_{i j}=w e^{\phi_{i j}} .
$$

Equation (14) is obviously invariant with respect to the discrete gauge transformation $w_{i j} \rightarrow w_{i j} e^{i\left(f_{i}-f_{j}\right)} ; \quad c_{j} \rightarrow c_{j} e^{i f_{j}}$. Therefore, any set of phases $\phi_{i j}$ satisfying Eqs. (12) can be used to find the spectrum and the eigenstates.

We have seen already that the problem of the quasiclassical spin is equivalent to the problem of a charged particle confined on the sphere $S^{2}$ in the homogeneous magnetic field of the monopole. It is a direct spherical analog to the problem of a charged particle moving on a plane in a homogeneous magnetic field, perpendicular to the plane. Restricting ourselves with the localized states, we consider a problem the planar analog of which is the problem of a charged particle on a two-dimentional (2D) lattice placed in a homogeneous magnetic field. It is known as the Harper equation [9]. The main difference from this famous problem studied by Harper, Azbel, Hofstadter, Thouless, Wiegmann, and many other authors $[9,10]$ is that, in our case the lattice is embedded in a sphere which is a compact manifold, in contrast to the planar case. Nevertheless, many features of the Harper equations will be encountered here, e.g., sudden variations in spectrum at a transition from a rational to an irrational flux through an elementary plaquette (see Sec. VI).

The initial Hamiltonian $\mathcal{H}(\mathbf{J})$ is assumed to possess a point group symmetry. It should be noted that $\mathcal{H}(\mathbf{J})$ is invariant with respect to the inversion transformation: $\mathbf{J} \rightarrow-\mathbf{J}$, whereas the reduced effective Hamiltonian is not. The reason is that this invariance which stems from the time-reversal symmetry cannot be extended onto the quantum permutation relations: $\left[J_{j}, J_{k}\right]=(\hbar / i) \epsilon_{j k l} J_{l}$. The time reversal requires also antilinear transformation of the state vectors [11] which cannot be incorporated into a linear symmetry group. Thus all groups of transformations under study consist of rotations only. The point groups in three dimensions have been studied thoroughly (see, for example, [5]). Special attention will be paid to the following point groups: $D_{n}, n=2,4,6, O$ (octahedron), and $Y$ (icosahedron).

In the next section we show that the action of the symmetry transformations on the effective Hamiltonian is not trivial due to Berry's phases.

\section{GROUP THEORY ANALYSIS}

\section{A. Construction of the main representation}

Let $\bar{G}$, a discrete subgroup of $\mathrm{SO}(3)$, be the point group of the crystal field, i.e., the point group leaving function $f(\mathbf{J})$ invariant. It always includes the space inversion $I$ as a consequence of the time-reversal symmetry. Also, we introduce a subgroup $G$ of the full symmetry group $\bar{G}=G \times C_{i}\left(C_{i}\right.$ $=\{E, I\})$ which includes rotation elements only. Further we employ the notation "symmetry group," namely, for $G$. Each group $G$ has several sets of equivalent symmetric directions defined by the intersection of equivalent $p$-fold sym- 
metry axes with the unit sphere. Let us denote such a configuration $\mathcal{C}(G, p)$ and corresponding number of symmetry directions $N(G, p)$ (we denoted it earlier as $N$ ). It can be readily seen that $N(G, p)=|G| / p$, where $|G|$ is the rank of the group $G$, i.e., the number of its elements. The set of $N$ $=N(G, p)$ localized states $|k\rangle$ corresponding to the configuration $C(G, p)$ is the vector space for a linear unitary representation of the group $G$. This representation depends also on $J$. Let us call it the main representation and denote it $W(G, p, J)$. Its dimensionality is obviously $N(G, p)$. For $J$ $=0, W(G, p, J)$ is a matrix representation of some subgroup $P$ of the permutation group $S_{N}$. Each element $g$ of $G$ can be put in one-to-one correspondence to a permutation $\mathcal{P}(g)$ $\in P$. The Hamiltonian of the system is invariant under their action. For $J \neq 0$ or $J$ not equivalent to 0 , the problem becomes quite peculiar since, due to Berry's phase factors, the Hamiltonian is no longer invariant under the action of the transformations $\mathcal{P}(g)$ :

$$
\mathcal{P H} \mathcal{P}^{T}=\mathcal{H}^{\prime} \neq \mathcal{H} .
$$

Hamiltonian $\mathcal{H}^{\prime}$ differs from $\mathcal{H}$ by a gauge transformation. Therefore, it is possible to append such a gauge transformation $\mathcal{U} \in U$ (unitary diagonal matrix) to each rotation that the Hamiltonian remains unchanged:

$$
\mathcal{U P H} \mathcal{P}^{T} \mathcal{U}^{\dagger}=\mathcal{H} .
$$

Thus, a proper representation $W(G, p, J)$ of the symmetry group for large spin $J$ or for Harper's equation on the sphere consists of operators $\mathcal{W}(g)=\mathcal{U}(g) \mathcal{P}(g)$. Since multiplication of each $\mathcal{W}(g)$ by an arbitrary phase factor does not violate Eq. (16), the matrices in $W(G, p, J)$ constitute a projective representation of $G$ in general, that is,

$$
\mathcal{W}\left(g_{1}\right) \mathcal{W}\left(g_{2}\right)=c\left(g_{1}, g_{2}\right) \mathcal{W}\left(g_{1} g_{2}\right), g_{1}, g_{2} \in G,
$$

where $c\left(g_{1}, g_{2}\right)$ is a function on $G \times G$ with values in $\mathrm{U}(1)$ (two-dimensional cochain).

Now, a question arises of whether the factor set $c\left(g_{1}, g_{2}\right)$ is equivalent to the trivial one: $c^{\prime}\left(g_{1}, g_{2}\right)=1$ for any $g_{1}, g_{2} \in G$, as it is for the case $J=0$. By definition, two factor sets $c$ and $c^{\prime}$ are equivalent if there exists a function $b(g)$ on $G$ with values in $\mathrm{U}(1)$ (one-dimensional cochain) such that

$$
c\left(g_{1}, g_{2}\right)=\frac{b\left(g_{1}\right) b\left(g_{2}\right)}{b\left(g_{1} g_{2}\right)} c^{\prime}\left(g_{1}, g_{2}\right) .
$$

We checked for finite groups $G \subset \mathrm{SO}(3)$ that Eq. (18) with $c^{\prime}\left(g_{1}, g_{2}\right)= \pm 1$ is really satisfied. In mathematical language this means that the cochain $c$ is a cocycle but not a coboundary for a half-integer spin and it is a coboundary for an integer spin [12]. Therefore, the factor set is nontrivial in general. It is equivalent to the multiplicative factors $\{ \pm 1\}$ (isomorphic to $\mathbf{Z}_{2}$ ) which is a consequence of the Dirac quantization: $2 J=n, n \in \mathbb{N}$. This structure of the factor set might have been anticipated since the parameter space of an arbitrary spin is not just $\mathrm{SO}(3)$ but its universal covering group $\mathrm{SU}(2)$ which can be obtained as a nontrivial extension of the former one: $1 \rightarrow \mathbf{Z}_{2} \rightarrow \mathrm{SU}(2) \rightarrow \mathrm{SO}(3) \rightarrow 1$. In our case, spin in crystal electric field (CEF), the proper group of sym- metries is $G$ extended by $\mathbf{Z}_{2}: 1 \rightarrow \mathbf{Z}_{2} \rightarrow \widetilde{G} \rightarrow G \rightarrow 1$. Instead of dealing with the projective representations of $G$, one can work with the linear representations of $\widetilde{G}$. An explicit construction of $\widetilde{G}$ will be given later in this section. In other language we must consider double-valued representations of $G$ [5] for half integer $J$.

The representation $W(G, p, J)$ was constructed for a particular gauge, however, one can easily find the required representation if the Hamiltonian undergoes a gauge transformation:

$$
\mathcal{U} \mathcal{H} \mathcal{U}^{\dagger}=\mathcal{H}^{\prime}
$$

Then a corrected representation leaves the Hamiltonian invariant:

$$
\mathcal{W}^{\prime}=\mathcal{U N U}^{\dagger}
$$

Harter and Patterson [2] used the Frobenius duality theorem [13] to find very elegantly the multiplicities of the low (high) energetic level clusters without using characters.

\section{B. Classification of configurations}

Generally speaking, the $N$-dimensional main representation is reducible. To perform the reduction of the main representation we need to find its characters. They are found explicitly in Appendix A. Here we issue final results. For $W(G, p, J)$, elements with non-zero characters are identity $E$, the rotation through an angle of $2 \pi$ about an arbitrary axis $Q$, and rotations $C_{p}^{q}$ about the $p$-fold axes.

$$
\begin{gathered}
\chi(E)=N, \quad \chi(Q)=N(-1)^{2 J}, \\
\chi\left(C_{p}^{q}\right)=2 \cos \left(\frac{2 \pi J q}{p}\right),
\end{gathered}
$$

$$
\chi\left(C_{p}^{q} Q\right)=2(-1)^{2 J} \cos \left(\frac{2 \pi J q}{p}\right), \quad(q=1, \ldots, p-1) .
$$

Now we proceed to consideration of different point groups and their configurations of extrema.

\section{Configurations of the octahedron group $O$}

Here, we classify possible configurations $\mathcal{C}(\mathbf{O}, p)$ of the octahedron symmetry group $\mathbf{O}$. In general, i.e., without accidental degeneracy, the minima (maxima) of the potential are located either on the equivalent symmetry axes of the cube or completely away from them (asymmetrically): $\mathcal{C}(\mathbf{O}, 4)$, three axes of the fourth order passing through the centers of opposite faces, $N=6 ; \mathcal{C}(\mathbf{O}, 3)$, four axes of the third order passing through opposite corners, $N=8 ; \mathcal{C}(\mathbf{O}, 2)$, six axes of the second order through the midpoints of opposite edges, $N=12 ; \mathcal{C}(\mathbf{O}, 1)$, none of the symmetry axes passes through the minima $N=24$ or $N=48$. The representations $W(\mathbf{O}, p, J)$ of the octahedron group acting on the spaces of states corresponding to the above described configurations are, respectively, 6, 8, 12, and 24(48) dimensional.

For a configuration $\mathcal{C}(\mathbf{O}, p)$ only elements $C_{p}^{q}$ have nonzero characters which were calculated earlier. They must be 
TABLE I. Irreducible components of the cubic representations $W_{N}, N=6,8,12,24$.

\begin{tabular}{lcccc}
\hline \hline$J$ & $\mathcal{C}(\mathbf{O}, 4)$ & $\mathcal{C}(\mathbf{O}, 3)$ & $\mathcal{C}(\mathbf{O}, 2)$ & $\mathcal{C}(\mathbf{O}, 1)$ \\
\hline 0 & $A_{1}, E, F_{1}$ & $A_{1}, A_{2}$ & $A_{1}, E$ & $A_{1}, A_{2}, E(2)$ \\
& & $F_{1}, F_{2}$ & $F_{1}, F_{2}(2)$ & $F_{1}(3), F_{2}(3)$ \\
1 & $F_{1}, F_{2}$ & $E, F_{1}, F_{2}$ & $A_{2}, E$ & \\
2 & $A_{2}, E, F_{2}$ & & $F_{1}(2), F_{2}$ & \\
$1 / 2$ & $E_{1}^{\prime}, G^{\prime}$ & $E_{1}^{\prime}, E_{2}^{\prime}, G^{\prime}$ & $E_{1}^{\prime}, E_{2}^{\prime}, G^{\prime}(2)$ & $E_{1}^{\prime}(2), E_{2}^{\prime}(2)$ \\
$3 / 2$ & $E_{2}^{\prime}, G^{\prime}$ & $G^{\prime}(2)$ & & $G^{\prime}(4)$ \\
\hline \hline
\end{tabular}

divided into classes of conjugate elements. The classes with nonzero characters (except $E$ and $Q$ ) are six rotations $C_{4}$ and $C_{4}^{3}$, and three rotations $C_{4}^{2}$ for $\mathcal{C}(\mathbf{O}, 4)$; eight rotations $C_{3}$ and $C_{3}^{2}$ for $\mathcal{C}(\mathbf{O}, 3)$; six rotations $C_{2}$ for $\mathcal{C}(\mathbf{O}, 2)$; and none for $\mathcal{C}(\mathbf{O}, 1)$.

The characters are periodic functions of $J$ with the period equal to $p$. This means that the multiplicities of the eigenvalues of the Hamiltonian have the same periodicity. The characters are invariant under the transformation $J \rightarrow-J(\bmod$ p) (reflection).

The irreducible components contained in representations $W_{N}, N=6,8,12,24$ of the octahedron group are given in Table I for values of $J$ inequivalent under the translations over $p$ and the reflection. These irreducible components, along with the components of $\mathbf{D}_{n}$ configurations, were found in [2]. For simplification we denoted $W(\mathbf{O}, p, J)$ as $W_{N}$, where $N=N(\mathbf{O}, p)$. The irreducible components of $W_{48}$ are not listed since there are twice as many of them as those for $W_{24}$. This relationship is correct for representation $W_{|\bar{G}|}$ of any group $G$. The characters of the accidental configurations, such as the 14-fold configurations on the boundary between $\mathcal{C}(\mathbf{O}, 4)$ and $\mathcal{C}(\mathbf{O}, 3)$, are merely sums of the characters of the constituting components and can be found from the given tables for the basic configurations.

\section{Configurations of the icosahedron group $Y$}

The classification of configurations $\mathcal{C}(\mathbf{Y}, p)$ for the icosahedron group of symmetries is similar to that of the octahedron group. Extrema can be located either along the directions of the symmetry axes or asymmetrically: $\mathcal{C}(\mathbf{Y}, 5)$, six axes of the fifth order passing through opposite corners of the icosahedron, $N=12 ; \mathcal{C}(\mathbf{Y}, 3)$, ten axes of the third order passing through the centers of opposite faces $N=20 ; \mathcal{C}(\mathbf{Y}, 2)$, 15 axes of the second order through the midpoints of opposite edges $N=30$; and $\mathcal{C}(\mathbf{Y}, 1)$, none of the symmetry axes passes through the minima $N=60$ or 120 . The main representations of the icosahedron group acting on the spaces of the configurations are, respectively, 12, 20, 30, and 60(120) dimensional. The classes with nonzero characters, besides $E$ and $Q$, are 12 rotations $C_{5}^{1,4}$ and 12 rotations $C_{5}^{2,3}$ for $\mathcal{C}(\mathbf{Y}, 5)$, 20 rotations $C_{3}^{1,2}$ for $\mathcal{C}(\mathbf{Y}, 3), 15$ rotations $C_{2}$ for $\mathcal{C}(\mathbf{Y}, 2)$, and none for $\mathcal{C}(\mathbf{Y}, 1)$. The multiplicities of the eigenvalues of the
TABLE II. Irreducible components of the icosahedron representations $W_{N}, N=12,20,30,60$.

\begin{tabular}{lcccc}
\hline \hline$J$ & $\mathcal{C}(\mathbf{Y}, 5)$ & $\mathcal{C}(\mathbf{Y}, 3)$ & $\mathcal{C}(\mathbf{Y}, 2)$ & $\mathcal{C}(\mathbf{Y}, 1)$ \\
\hline & $A, F_{1}$ & $A, F_{1}, F_{2}$ & $A, F_{1}, F_{2}$ & $A, F_{1}(3), F_{2}(3)$ \\
& & & & \\
& $F_{2}, H$ & $G(2), H$ & $G(2), H(3)$ & $G(4), H(5)$ \\
1 & $F_{1}, G, H$ & $F_{1}, F_{2}$ & $F_{1}(2), F_{2}(2)$ & \\
& & & & \\
2 & $F_{2}, G, H$ & & & \\
& & $E_{1}^{\prime}, E_{2}^{\prime}$ & $E_{1}^{\prime}, E_{2}^{\prime}$ & $E_{1}^{\prime}(2), E_{2}^{\prime}(2)$ \\
$1 / 2$ & $E_{1}^{\prime}, G^{\prime}, I^{\prime}$ & & & \\
& & $G^{\prime}, I^{\prime}(2)$ & $G^{\prime}(2), I^{\prime}(3)$ & $G^{\prime}(4), I^{\prime}(6)$ \\
$3 / 2$ & $E_{2}^{\prime}, G^{\prime}, I^{\prime}$ & $G^{\prime}(2), I^{\prime}(2)$ & & \\
$5 / 2$ & $I^{\prime}(2)$ & & & \\
\hline \hline
\end{tabular}

Hamiltonian, for a configuration $\mathcal{C}(\mathbf{Y}, p)$, have the period $p$. The irreducible components contained in representations $W_{N}, N=12,20,30,60$ of the icosahedron group are given in Table II.

\section{Configurations of $\mathrm{D}_{2}$}

Next, we consider the configurations of three groups of symmetries $\mathbf{D}_{N}(N=2,4,6)$. Despite their simplicity, Berry's phase introduces here some interesting effects as well.

The configurations of $\mathbf{D}_{2}$ are quite simple $\mathcal{C}\left(\mathbf{D}_{2}, 2\right)$, one axis of the second order, $N=2$; and $\mathcal{C}\left(\mathbf{D}_{2}, 1\right)$, none of the symmetry axes passes through the extrema, $N=4$ or 8 The characters of the $\mathbf{D}_{2}$ representations and the irreducible components contained in representations $W_{N}, N=2,4$ are given in Table III.

\section{Configurations of the tetragonal group $\mathrm{D}_{4}$}

The configurations of $\mathbf{D}_{4}$ are $\mathcal{C}\left(\mathbf{D}_{4}, 4\right)$, one axis of the fourth order, $N=2 ; \mathcal{C}\left(\mathbf{D}_{4}, 2\right)$, two axes of the second order, $N=4$; and $\mathcal{C}\left(\mathbf{D}_{\mathbf{4}}, 1\right)$ none of the symmetry axes passes through the extrema, $N=8$ or 16 . The irreducible components contained in representations $W_{N}, N=2,4,8$ of $\mathbf{D}_{4}$ are given in Table IV.

An interesting conclusion can be drawn from the data in Table IV. The twofold classical degeneracy of the configurations of $\mathcal{C}\left(\mathbf{D}_{4}, 4\right)$ is not lifted for all but even values of $J$. Thus, the tunneling is allowed only for even spins. This result cannot be accounted for by Kramers degeneracy, as was possible in [14] for $\mathbf{D}_{2}$ configuration, and is totally due to the symmetry combined with Berry's phase. Also, it shows importance of the details of the background, i.e., $\mathbf{D}_{2}$ (considered in the preceding section and in [14]), $\mathbf{D}_{4}$, and $\mathbf{D}_{6}$ (considered in the next section) groups of symmetries have the

TABLE III. Irreducible components of the $\mathbf{D}_{2}$ representations.

\begin{tabular}{lcc}
\hline \hline$J$ & $\mathcal{C}\left(\mathbf{D}_{2}, 2\right)$ & $\mathcal{C}\left(\mathbf{D}_{2}, 1\right)$ \\
\hline 0 & $A, B_{3}$ & $A, B_{1}, B_{2}, B_{3}$ \\
1 & $B_{1}, B_{2}$ & \\
$1 / 2$ & $E^{\prime}$ & $E^{\prime}(2)$ \\
\hline \hline
\end{tabular}


TABLE IV. Irreducible components of the $\mathbf{D}_{4}$ representations.

\begin{tabular}{lccc}
\hline \hline$J$ & $\mathcal{C}\left(\mathbf{D}_{4}, 4\right)$ & $\mathcal{C}\left(\mathbf{D}_{4}, 2\right)$ & $\mathcal{C}\left(\mathbf{D}_{4}, 1\right)$ \\
\hline 0 & $A_{1}, A_{2}$ & $A_{1}, B_{1}, E$ & $A_{1}, A_{2}, B_{1}, B_{2}, E(2)$ \\
1 & $E$ & $A_{2}, B_{2}, E$ & \\
& $B_{1}, B_{2}$ & & \\
$1 / 2$ & $E_{1}^{\prime}$ & $E_{1}^{\prime}, E_{2}^{\prime}$ & $E_{1}^{\prime}(2), E_{2}^{\prime}(2)$ \\
$3 / 2$ & $E_{2}^{\prime}$ & & \\
\hline \hline
\end{tabular}

easy axis (twofold) configuration, however, the tunneling is allowed in the $\mathbf{D}_{N}$ environment only for $J=0(\bmod N / 2)$ and is defined by the anisotropy in the plane normal to the easy axis.

\section{Configurations of the hexagonal group $D_{6}$}

Due to the similarity of this group with $\mathbf{D}_{4}$, we just present the data on the $\mathbf{D}_{6}$ representations. $\mathcal{C}\left(\mathbf{D}_{6}, 6\right)$, one axis of the sixth order, $N=2 ; \mathcal{C}\left(\mathbf{D}_{6}, 2\right)$, three axes of the second order, $N=6$; and $\mathcal{C}\left(\mathbf{D}_{6}, 1\right)$, none of the symmetry axes passes through the minima, $N=12$ or 24 . See Table V.

\section{SPECTRUM}

The group-theoretical analysis of the preceding section gives the number of split sublevels in the initial $\mathrm{N}$-fold multiplet and their degeneracies. In this section we find the order of the sublevels and distances between them. It requires explicit diagonalization of the reduced Hamiltonian. As we show below, the spectrum is a much more subtle matter than the number and degeneracy of the sublevels. It may depend on details of the Hamiltonian.

We assume that all tunneling paths between nearest minima are equivalent, that is, all nonzero tunneling amplitudes have equal absolute values $|w|$. Consequently, $w$ enters the Hamiltonian as a common multiplier and all eigenvalues are multiples of $w$ in zero magnetic field. The solid angle covered by the minimal nontrivial closed path will be assumed known. It is, actually, a constant for all configurations but $\mathcal{C}(G, 2), G=\mathbf{O}, \mathbf{Y}$, where it is a function of some dimensionless combinations of the CEF parameters, e.g., ratio $u$ in Sec. II.

In some cases, not only in simple ones, such as $\mathcal{C}\left(\mathbf{D}_{N}, N\right)$, there are two tunneling trajectories connecting nearest minima. E.g., in the vicinity of the boundary between sixfold and eightfold configurations of the cubic group (see Fig. 3), the tunneling trajectory deviates from the geodesics connecting the minima and, due to the symmetry, there are two

TABLE V. Irreducible components of the $\mathbf{D}_{6}$ representations.

\begin{tabular}{lccc}
\hline \hline$J$ & $\mathcal{C}\left(\mathbf{D}_{6}, 6\right)$ & $\mathcal{C}\left(\mathbf{D}_{6}, 2\right)$ & $\mathcal{C}\left(\mathbf{D}_{6}, 1\right)$ \\
\hline 0 & $A_{1}, A_{2}$ & $A_{1}, B_{1}, E_{1}, E_{2}$ & $A_{1}, A_{2}, B_{1}, B_{2}, E_{1}(2), E_{2}(2)$ \\
1 & $E_{1}$ & $A_{2}, B_{2}, E_{1}, E_{2}$ & \\
2 & $E_{2}$ & & \\
3 & $B_{1}, B_{2}$ & & \\
$1 / 2$ & $E_{1}^{\prime}$ & $E_{1}^{\prime}, E_{2}^{\prime}, E_{3}^{\prime}$ & $E_{1}^{\prime}(2), E_{2}^{\prime}(2), E_{3}^{\prime}(2)$ \\
$3 / 2$ & $E_{3}^{\prime}$ & & \\
$5 / 2$ & $E_{2}^{\prime}$ & & \\
\hline \hline
\end{tabular}

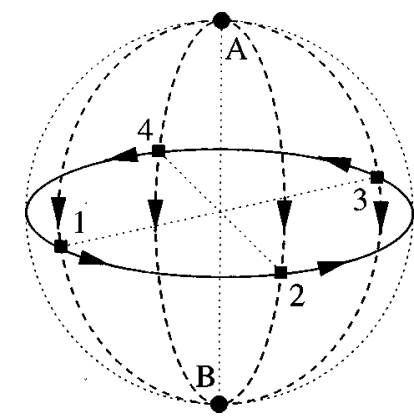

FIG. 4. Configurations of $\mathbf{D}_{4}$. Filled circles and dashed lines belong to $\mathcal{C}\left(\mathbf{D}_{4}, 4\right)$, and filled squares and solid lines belong to $\mathcal{C}\left(\mathbf{D}_{4}, 2\right)$ respectively.

trajectories located symmetrically with respect to the geodesics. However, the two trajectories can be considered as one effective path with the tunneling amplitude of $2 w \cos (J \Omega / 2)$ [see Eq. (28)], where $w$ is the tunneling amplitude of a single path and $\Omega$ is the solid angle subtended by the two trajectories.

Before proceeding to a detailed analysis of the spectra, we obtain some relations between eigenvalues of the same configuration, but for different $J$. These relations are of purely geometric origin [15]. Let us assume that the parameter space $S^{2}$ can be covered completely and without overlap by $s$ congruent plaquettes whose boundaries are the tunneling trajectories. E.g., these are two hemispheres for $\mathcal{C}\left(\mathbf{D}_{N}, 2\right), N$ $=2,4,6$, configurations; $N$ orangelike segments for $\mathcal{C}\left(\mathbf{D}_{N}, N\right)$, Fig. 4; eight curved right-angled triangles for $\mathcal{C}(\mathbf{O}, 4)$, Fig. 1. Each plaquette subtends a solid angle of $4 \pi / s$, and Berry's phase for each loop is $4 \pi J / s$. Then, from Eq. (12), it follows that the spectrum is a periodic function of $J$ with the period $s / 2{ }^{1}$ The spectra of systems differing by transformation $\gamma(c) \rightarrow-\gamma(c)$ must be identical due to the time-reversal symmetry. Hence, all $J$ 's are divided into $s / 2+1$ equivalence classes defined by a set of numbers $0,1 / 2,1, \ldots, s / 4$. A fixed $J$ belongs to the class of equivalence labeled by

$$
\min _{n \in \mathbb{Z}}|J+n s / 2|
$$

Hereinafter, we will work only with the minimal nonequivalent $J$ 's.

In a more general setting, i.e., in the presence of $n$ different elementary plaquettes, periodicity of the spectra depends on the rationality of the flux quanta passing through each plaquette: if a flux per each plaquette is $\Phi_{i}=J \Omega_{i}$ $=2 \pi J P_{i} / Q_{i}, i=1, \ldots, n$, where $P_{i}$ and $Q_{i}$ are mutually prime integers, then the period of the spectra is the least common multiple of $Q_{i}, i=1, \ldots, n$. Otherwise the spectra are not periodic and each $J$ represents a class. Thus, if $n$ $=1$ the spectra is always periodic and if $n>1$ it is not in general (unless an additional symmetry is present).

\footnotetext{
${ }^{1}$ This statement is conventional; it is periodic if $w$ does not depend on $J$. However, the ratios of the interlevel distances are periodic functions of $J$.
} 
TABLE VI. Spectrum of $\mathcal{C}\left(\mathbf{D}_{4}, 2\right)$ in magnetic field, the limit of small magnetic field, and low-temperature magnetic susceptibility $\left[\beta=1 /\left(k_{B} T\right)\right]$.

\begin{tabular}{lcc}
\hline \hline$J$ & Eigenvalues & Susceptibility \\
\hline 0 & $\pm\left(2 w+\frac{1}{4} h^{2} J^{2} / w\right), \pm \frac{1}{4} h^{2} J^{2} \sin \left(2 \phi_{h}\right) / w$ & $\frac{1}{2}\left(g J \mu_{B}\right)^{2} / w$ \\
$1 / 2$ & $\pm\left(\sqrt{2} w \pm \frac{1}{2} h J+\frac{\sqrt{2}}{16} h^{2} J^{2} / w\right)$ & $\frac{1}{4}\left(g J \mu_{B}\right)^{2} \beta$ \\
\hline \hline
\end{tabular}

An extra symmetry of the spectra can be extracted by considering an operation of the change of sign: $w \rightarrow-w$. This transformation inverts energy levels inside of each class. On the other hand, the spectra depend only on gauge invariants $w^{k} \cos \left(J \Omega_{k}\right)$, where $\Omega_{k}=4 m \pi / s \quad$ ( $m$ is an integer) is the solid angle subtended by a closed contour containing $k$ tunneling paths and is a multiple of the solid angle subtended by the elementary plaquette $4 \pi / s$. If all closed contours contain an even number of paths ( $k$ is even), e.g., $\mathcal{C}(\mathbf{O}, 3)$, $\mathcal{C}\left(\mathbf{D}_{4}, 2\right)$, the levels are symmetric inside of each class, that is, they come in pairs of opposite sign $\pm E$. For example, for eigenvalues of $\mathcal{C}(\mathbf{O}, 3)$ the following relations are satisfied: $E\left(J=0 ; A_{1}\right)=-E\left(J=0 ; A_{2}\right), \quad E\left(J=0,1 ; F_{1}\right)=-E(J$ $\left.=0,1 ; F_{2}\right), \quad E(J=1 ; E)=0, \quad E\left(J=1 / 2 ; E_{1}^{\prime}\right)=-E(J$ $\left.=1 / 2 ; E_{2}^{\prime}\right), E\left(J=1 / 2 ; G^{\prime}\right)=0$. If some of the closed contours consist of an odd number of paths, e.g., $\mathcal{C}(\mathbf{O}, 4), \mathcal{C}(\mathbf{Y}, 5)$, then the simultaneous change of sign $w \rightarrow-w$ and shift $J \rightarrow J$ $+s / 4$ leaves the invariant combinations unchanged. Therefore, each level $E$ in the class of $J$ has its counterpart $-E$ in the class of $J+s / 4$. For example, in $\mathcal{C}(\mathbf{O}, 4): E\left(J=0 ; A_{1}\right)=$ $-E\left(J=2 ; A_{2}\right), E\left(J=0 ; F_{1}\right)=-E\left(J=2 ; F_{2}\right), E(J=0 ; E)=$ $-E(J=2 ; E), \quad E\left(J=1 / 2 ; E_{1}^{\prime}\right)=-E\left(J=3 / 2 ; E_{2}^{\prime}\right), \quad E(J$ $\left.=1 / 2 ; G^{\prime}\right)=-E\left(J=3 / 2 ; G^{\prime}\right)$. If $J$ and $J+s / 4$ belong to the same equivalence class, their spectrum is symmetric, e.g., in $\mathcal{C}(\mathbf{O}, 4): E\left(J=1 ; F_{1}\right)=-E\left(J=1 ; F_{2}\right)$.

The analysis carried out by Harter and Patterson [2] led to similar results for some configurations, e.g., $\mathcal{C}\left(\mathbf{D}_{n}, 2\right)$, $\mathcal{C}(\mathbf{O}, 4)$, and $\mathcal{C}(\mathbf{O}, 3)$, but without magnetic field.

Later in this section we calculate the spectra for different groups of symmetry and configurations.

\section{A. Spectra of the $D_{n}(n=2,4,6)$}

The configurations of $\mathbf{D}_{4}$ are shown in Fig. 4. In the case of the $\mathbf{D}_{6}$ configurations, there are six minima on the equatorial circle $\left[\mathcal{C}\left(\mathbf{D}_{6}, 2\right)\right]$ and six tunneling paths connecting the antipodal points $\left[\mathcal{C}\left(\mathbf{D}_{6}, 6\right)\right]$. For $\mathcal{C}\left(\mathbf{D}_{2}, 2\right)$, it is just two minima connected by two tunneling trajectories. The total tunneling amplitude for $\mathcal{C}\left(\mathbf{D}_{N}, N\right)$, from one pole to the other, is

$$
w \sum_{k=0}^{N-1} \exp (i 4 \pi k J / N)
$$

where we prescribed a phase factor of unity to one of the tunneling paths. The Hamiltonian is a $2 \times 2$ matrix with the following eigenvalues:
TABLE VII. Spectrum of $\mathcal{C}\left(\mathbf{D}_{6}, 2\right)$ in magnetic field, the limit of small magnetic field, and low temperature magnetic susceptibility.

\begin{tabular}{ccc}
\hline \hline$J$ & Eigenvalues & Susceptibility \\
\hline & $\pm\left(2 w+\frac{1}{2} h^{2} J^{2} / w\right)$ & \\
0 & $\pm\left(w-\frac{3}{8} h^{2} J^{2} / w\right)$ & $\left(g J \mu_{B}\right)^{2} / w$ \\
& $\pm\left(w+\frac{1}{8} h^{2} J^{2} / w\right)$ & \\
$1 / 2$ & $0(2), \pm\left(\sqrt{3} w \pm \frac{1}{2} h J+\frac{\sqrt{3}}{12} h^{2} J^{2} / w\right)$ & $\frac{1}{4}\left(g J \mu_{B}\right)^{2} /\left(k_{B} T\right)$ \\
\hline \hline
\end{tabular}

$$
E=\left\{\begin{array}{l} 
\pm 2 w \cos (\pi J) \text { for } N=2 \\
\pm 4 w \cos (\pi J) \cos (\pi J / 2) \text { for } N=4 \\
\pm 2 w \cos (\pi J)[1+2 \cos (2 \pi J / 3)] \text { for } N=6
\end{array}\right.
$$

In full agreement with the predictions of Sec. IV, the paths interfere destructively for all spin values except $J=0(\bmod$ $N / 2)$.

The case of minimal symmetry $\mathcal{C}\left(\mathbf{D}_{2}, 2\right)$ has been considered by Loss et al. [14] earlier. They argued that in the case of half integer $J$ the tunneling amplitudes along the two paths cancel each other. One can see from Eqs. (22) that, when the number of equivalent tunneling paths increases due to the symmetry, such a cancellation takes place for integer $J$ as well [with the exception of $J=0(\bmod N / 2)]$, where the classical degeneracy of the ground-state level is twofold for all $\mathcal{C}\left(\mathbf{D}_{N}, N\right)$.

In the presence of magnetic field the eigenvalues are $E(h)= \pm \sqrt{E^{2}(0)+(h J)^{2}}$, where $h=g \mu_{B} H$ and $H$ is the component of magnetic field along the easy direction.

For the $\mathcal{C}\left(\mathbf{D}_{N}, 2\right) N=4,6$ configurations, the Hamiltonian is that of the one-dimensional $N$-site tight-binding model [7], with eigenvalues. ${ }^{2}$

$$
E_{k}=2 w \cos [2 \pi(k+J) / N], \quad k=0,1, \ldots, N-1 .
$$

The magnetic field enters the Hamiltonian as a sitediagonal matrix:

$$
\mathcal{H}_{h}=-h J \cos \left(\phi_{h}-2 \pi l / N\right), \quad l=0,1, \ldots, N-1,
$$

where $h=g \mu_{B} H, H$ is the in-plane component of magnetic field, and $\phi_{h}$ is the angle of this component with respect to the easy direction of the CEF labeled by $l=0$. The eigenvalues of $\mathcal{H}+\mathcal{H}_{h}$ can be found analytically. For $\mathcal{C}\left(\mathbf{D}_{4}, 2\right)$, one finds

$$
E^{2}=2 w^{2}+\frac{\bar{h}^{2}}{2} \pm \sqrt{4 w^{4} \cos ^{2}(\pi J)+2 \bar{h}^{2} w^{2}+\frac{\bar{h}^{4}}{4} \cos ^{2}\left(2 \phi_{h}\right)}
$$

where we used $\bar{h}$ as a shorthand for $h J$. The spectra of $\mathcal{C}\left(\mathbf{D}_{4}, 2\right)$ (previously calculated in $\left.[7]\right)$ and $\mathcal{C}\left(\mathbf{D}_{6}, 2\right)$ in magnetic field are given in the limit of small magnetic fields in Tables VI and VII, respectively. The last column of Tables VI, VII is the low-temperature magnetic susceptibility,

\footnotetext{
${ }^{2}$ The label $k$ in Eq. (23) does not correspond to the algebraic value of the level.
} 
TABLE VIII. Spectra and low-temperature magnetic susceptibilities of $\mathcal{C}(\mathbf{O}, 4)\left[\beta=1 /\left(k_{B} T\right)\right.$, a common factor of $\left(g J \mu_{B}\right)^{2}$, is omitted].

\begin{tabular}{lcc}
\hline \hline \multicolumn{1}{c}{$J$} & Eigenvalues (degeneracies) & Susceptibility \\
\hline 0 & $-2 w(2), 0(3), 4 w(1)$ & $1 /(3 w)$ \\
1 & $-2 w(3), 2 w(3)$ & $\beta / 6$ \\
2 & $-4 w(1), 0(3), 2 w(2)$ & $1 /(6 w)$ \\
$1 / 2$ & $-\sqrt{2} w(4), 2 \sqrt{2} w(2)$ & $2 \beta / 9$ \\
$3 / 2$ & $-2 \sqrt{2} w(2), \sqrt{2} w(4)$ & $\beta / 9$ \\
\hline
\end{tabular}

which is a readily observable physical quantity. The susceptibility saturates to a constant for the classes without a magnetic moment in the ground state (integer spins) and has a Curie-like behavior for the ones with a magnetic moment in the ground state (half-integer spins); see Appendix C for details.

In the case of $\mathcal{C}\left(\mathbf{D}_{N}, 2\right)$ configurations, there is a spectral difference between integer and half-integer spins only which can be ascribed to Kramers degeneracy. In the next section, we consider non-Abelian cases, where more complex division on equivalence classes occurs.

\section{B. Spectra of the $\mathbf{O}$ configurations}

The cubic symmetries are quite common in nature. We will perform a detailed study of the configurations of the octahedron group. The Hamiltonian for configuration $\mathcal{C}(\mathbf{O}, 4)$ has the following matrix elements:

$$
\begin{gathered}
h_{i i}=0, \quad i=1,2, \ldots, 6 \\
h_{i j}=0, \quad|i-j|=1, \quad i+j=3,7,11 \\
\left|h_{i j}\right|=|w| \text { for other } 1 \leqslant i, j \leqslant 6,
\end{gathered}
$$

where we adopted the enumeration shown in Fig. 1. The tunneling trajectories divide the sphere into eight plaquettes. Relation (12), written for each plaquette, gives eight equations for the phases $\phi_{i j}$, where $\Omega(c)=\pi / 2$ (an example of a set of the phases for this configuration as well as a calculation of the spectra is given in Appendix B). Only seven equations are independent. Given definite phases, the diagonalization is straightforward. The eigenvalues can be expressed in the following closed form [15]:

$$
\begin{gathered}
E_{k}(J)=(-1)^{k} 2 w \chi[\pi(J+2 k)], k=0, \ldots, 5 \\
\chi(x)=\cos \frac{2 x}{3} \cos \frac{x}{2}-\left(\cos ^{2} \frac{x}{3}+\sin ^{2} \frac{2 x}{3} \sin ^{2} \frac{x}{2}\right)^{1 / 2} .
\end{gathered}
$$

The ordered spectra of $\mathcal{C}(\mathbf{O}, 4)$ are given in Table VIII ( $w$ $>0$ ) for the minimal set of J's; the spectra for other $J$ 's can be obtained by the use of the equivalence relation (20). Note that the spectra should be inverted if $w$ is negative.

The physical difference among the classes is manifested when magnetic field is applied. The magnetic part of the Hamiltonian in this case is

$$
\mathcal{H}_{h}=J \operatorname{diag}\left(-h_{z}, h_{z},-h_{x}, h_{x},-h_{y}, h_{y}\right) .
$$

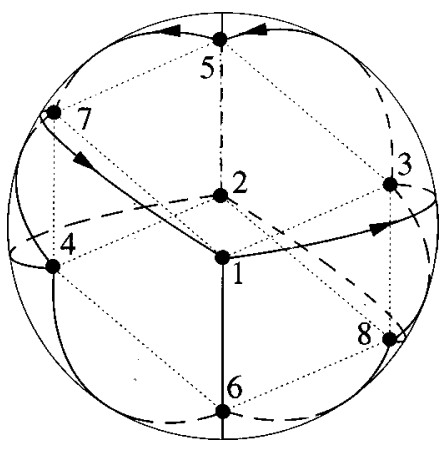

FIG. 5. Paths of the spin on the unit sphere between the easy positions of the field. The case of $\mathcal{C}(\mathbf{O}, 3)$ configuration.

The full Hamiltonian can be easily diagonalized for some symmetric direction of the field, e.g., along easy direction $(1,0,0)$. The direction of the field does not influence the lowtemperature susceptibility since the latter is isotropic in a cubic CEF. However, individual levels of the ground-state multiplet may have anisotropic magnetic susceptibility as well as anisotropic magnetization. The low-temperature magnetic susceptibilities of $\mathcal{C}(\mathbf{O}, 4)$ are collected in the last column of Table VIII.

In the case of the $\mathcal{C}(\mathbf{O}, 3)$ configuration, the minima are located at the vertices of a cube inscribed into the unit sphere: $\sin \theta=\sqrt{2 / 3}, \sin (2 \phi)=0$, where $\theta$ and $\phi$ are the spherical coordinates of the minima (see Fig. 5). The tunneling trajectories divide the surface of the sphere into six congruent plaquettes; each subtends a solid angle of $2 \pi / 3$. Five independent equations (12) fix the tunneling phase shifts and the Hamiltonian up to an arbitrary gauge transformation. The eight eigenvalues are [15]

$$
\begin{gathered}
E_{k}^{ \pm}= \pm 2 w \xi[\pi(J+3 k)], \quad k=0,1,2,3, \\
\xi(x)=\left(3+2 \cos x \cos \frac{2 x}{3}+4 \cos \frac{x}{2} \cos \frac{x}{3} \varrho(x)\right)^{1 / 2}, \\
\varrho(x)=\left(4 \sin ^{2} \frac{x}{2} \sin ^{2} \frac{x}{3}+1\right)^{1 / 2} .
\end{gathered}
$$

The ordered eigenvalues are presented in Table IX for the nonequivalent $J$ 's $(w>0)$. Analysis of the magnetic response is quite straightforward as well (see Appendix C); the magnetic susceptibilities of the classes are given in the last column of Table IX.

Consideration of $\mathcal{C}(\mathbf{O}, 2)$ will be postponed until Sec. VI.

TABLE IX. Spectra and low-temperature magnetic susceptibilities of $\mathcal{C}(\mathbf{O}, 3) \quad\left[\beta=1 /\left(k_{B} T\right)\right.$, a common factor of $\left(g J \mu_{B}\right)^{2}$, is omitted].

\begin{tabular}{lcc}
\hline \hline \multicolumn{1}{c}{$J$} & Eigenvalues (degeneracies) & Susceptibility \\
\hline 0 & $-3 w(1),-w(3), w(3), 3 w(1)$ & $1 /(3 w)$ \\
1 & $-2 w(3), 0(2), 2 w(3)$ & $\beta / 6$ \\
$1 / 2$ & $-\sqrt{6} w(2), 0(4), \sqrt{6} w(2)$ & $\beta / 9$ \\
$3 / 2$ & $-\sqrt{3} w(4), \sqrt{3} w(4)$ & $2 \beta / 9$ \\
\hline \hline
\end{tabular}




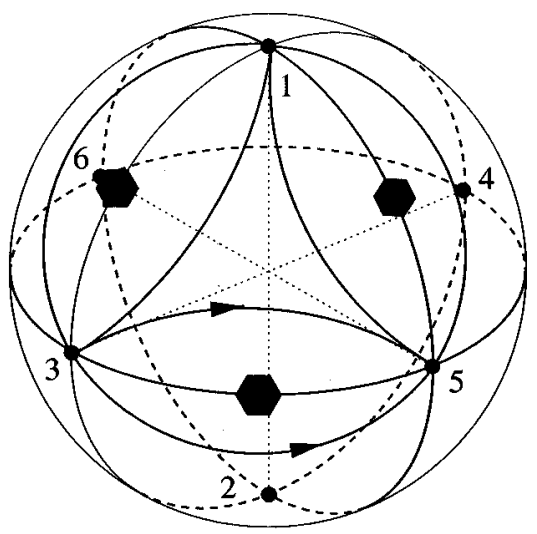

FIG. 6. Tunneling trajectories of the spin (double paths). The sixfold configurations of $\mathbf{O}$. The hexagons show the locations of the maxima of the CEF potential.

\section{Multiple tunneling path regime}

In the $\mathcal{C}(\mathbf{O}, 4)$ configuration, a tunneling trajectory connecting two minima, e.g., minima 3 and 5 in Fig. 1, is not necessarily a geodesic on the sphere. For example, if the midpoint of the geodesics connecting minima 3 and 5 is a maximum of the CEF potential then the tunneling trajectory connecting the minima will split in two paths: one deviating towards the "north" pole (minimum 1) and the other towards the "south" pole (minimum 2) as is shown in Fig. 6. One path is a mirror copy of the other with respect to the "equatorial" plane. Thus, the absolute values of the tunneling amplitudes corresponding to the two trajectories $(|w|)$ are identical. To find the compound tunneling amplitude we assume that one of the trajectories, e.g., the one connecting minima 3 and 5, and located in the "south" hemisphere, has the phase $\varphi_{1}: w_{1}=|w| \exp \left(i \varphi_{1}\right)$. Then, due to the Berry connection, the other amplitude must be $w_{2}=|w| \exp \left[i\left(\varphi_{1}\right.\right.$ $-J \Omega)$, where $\Omega$ is the solid angle subtended by the two trajectories. The effective amplitude is

$$
w_{e}=w_{1}+w_{2}=2|w| e^{i\left(\varphi_{1}-J \Omega / 2\right)} \cos (J \Omega / 2) .
$$

Interesting conclusions can be derived from formula (28). First, the splitting of the trajectories does not change the connectivity matrix of the configuration, it just modifies the multiplier of Hamiltonian (25) and all results obtained for configuration $\mathcal{C}(\mathbf{O}, 4)$ hold true. Secondly, the spectrum may be an oscillating function of $J$ or, if one were able to vary parameters in such a way that $\Omega$ changes from its maximum value to zero, several oscillations of the spectrum could be observed as well. To estimate the number of oscillations we use the fact that different tunneling trajectories emanating from a site and ending at some other site(s) do not intersect at intermediate points (they can only intersect at the end points). Then we can state that the maximal possible deviation of the trajectories from the spherical geodesics connecting the positions of the $\mathcal{C}(\mathbf{O}, 4)$ configuration is reached when the trajectories pass along the spherical geodesics connecting the geometrically closest positions of the $\mathcal{C}(\mathbf{O}, 3)$ and $\mathcal{C}(\mathbf{O}, 4)$ configurations. Figure 7 depicts this situation: the two tunneling trajectories connecting the sixfold global minima 3 and 5 (filled circles) are passing very closely to the eightfold

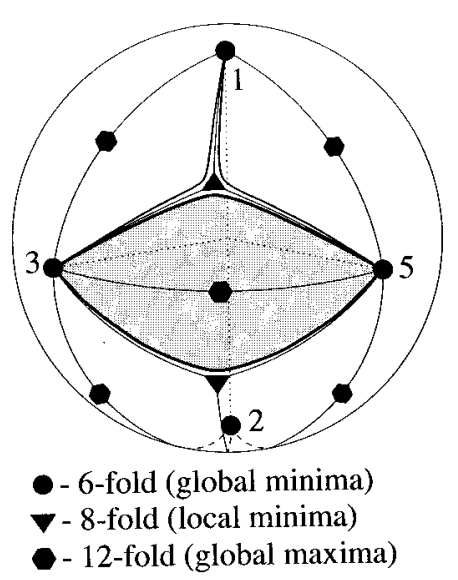

FIG. 7. Tunneling trajectories of the spin (double paths). The sixfold configurations of $\mathbf{O}$. The tunneling trajectories pass closely to the local minima (locations of the eightfold configuration).

local minima (filled triangles), thus "avoiding' the 12-fold global maxima (filled hexagons). The solid angle enclosed by the two trajectories (shaded area in Fig. 7) varies in the range $0 \leqslant \Omega<\pi / 3$. Upon such a variation of $\Omega$ the spectrum will make $J / 12$ full oscillations.

Next we analyze the multiple tunneling trajectories of $\mathcal{C}(\mathbf{O}, 3)$. Figure 8 depicts the splitting of the trajectory connecting minima 1 and 5 (solid curves $A$ and $B$ ). The situation is similar to that of the $\mathcal{C}(\mathbf{O}, 4)$ configuration (the oscillations take place and their maximal number is $J / 12$ ) except one subtle point: when a trajectory deviates strongly from the geodesics it approaches the trajectory connecting a nextnearest-neighbor (dashed lines in Fig. 8), e.g., lines $A^{\prime}$ and $B^{\prime}$ which connect 1 with 4 and 8 , respectively. This is a very drastic change in the tunneling regime which leads to a change of the connectivity matrix.

To calculate the spectrum we assume that the absolute values of the single tunneling amplitudes to the nearest- and next-nearest-neighbor sites are the same $w$. However, the effective amplitude for the nearest-neighbor tunneling is $2 w \cos (J \Omega / 2)$ due to the double trajectories. The elementary plaquette, in this case, is a triangle covering the solid angle of $\pi / 3$, e.g., triangle 1-5-8-1 in Fig. 8. In this case, plaquettes cover the sphere twice. Then, the periodicity of the spectra is

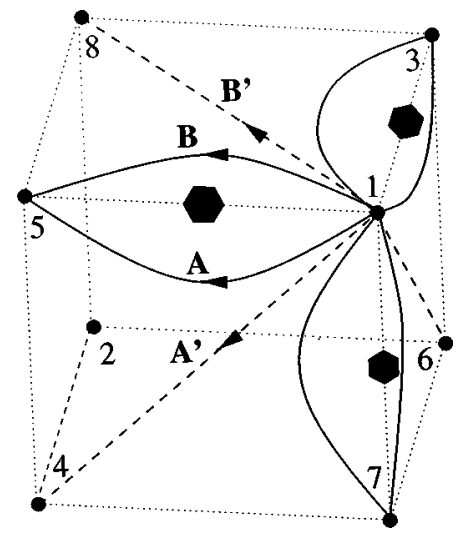

FIG. 8. Tunneling trajectories of the spin (double paths). The eightfold configurations of $\mathbf{O}$. The hexagons show the locations of the maxima of the CEF potential. 
TABLE X. Spectra of $\mathcal{C}(\mathbf{O}, 3)$, region of the multiple tunneling path regime $[x=2 \cos (J \Omega / 2)]$; all eigenvalues are multiples of $w$.

\begin{tabular}{lc}
\hline \hline$J$ & Eigenvalues (degeneracies) \\
\hline 0 & $-3(1-x)(1),-(1+x)(3),(1-x)(3), 3(1+x)(1)$ \\
1 & $-2(1-x / 2)(3),-3 x(2), 2(1+x / 2)(3)$ \\
2 & $-2(1+x / 2)(3), 3 x(2), 2(1-x / 2)(3)$ \\
3 & $-3(1+x)(1),-(1-x)(3),(1+x)(3), 3(1-x)(1)$ \\
$1 / 2$ & $-(\sqrt{6}-x \sqrt{3})(2),-x \sqrt{3}(4),(\sqrt{6}+x \sqrt{3})(2)$ \\
$3 / 2$ & $-\sqrt{3\left(1+x^{2}\right)}(4), \sqrt{3\left(1+x^{2}\right)}(4)$ \\
$5 / 2$ & $-(\sqrt{6}+x \sqrt{3})(2), x \sqrt{3}(4),(\sqrt{6}-x \sqrt{3})(2)$ \\
\hline \hline
\end{tabular}

given by $s=4 \pi /(\pi / 3)$, which is half of the total number of the elementary plaquettes. Application of the symmetry arguments given at the beginning of this section leads to the following properties of the spectrum: the periodicity of the spectral behavior is $J=s / 2=6,|J+6 n|, n \in \mathbb{Z}$ is equivalent to $J$, the spectrum of $J+3$ is the inverted spectrum of $J$. The results of the diagonalization are summarized in Table X.

\section{Spectra of the $Y$ configurations}

The analysis of the configurations of the $\mathbf{Y}$ group is tedious, though similar to that for the $\mathbf{O}$ group. We present only the results of the analysis here. Table XI contains the spectra and the low-temperature susceptibilities of the $\mathcal{C}(\mathbf{Y}, 5)$ configuration (the energies are multiples of $w$ ). Tables XII, XIII contain the spectra and the low-temperature susceptibilities of the $\mathcal{C}(\mathbf{Y}, 3)$ configuration, respectively.

The multiple tunneling path regime is present in configurations $\mathcal{C}(\mathbf{Y}, 5)$ and $\mathcal{C}(\mathbf{Y}, 3)$ as well. Its analysis is similar to that of configurations $\mathcal{C}(\mathbf{O}, 4)$ and $\mathcal{C}(\mathbf{O}, 3)$. We present here its summary only: The regions of existence of configurations $\mathcal{C}(\mathbf{Y}, 5)$ and $\mathcal{C}(\mathbf{Y}, 3)$ in the parameter space of the CEF are divided into two parts for each configuration. One part corresponds to the single tunneling path regime. The above theory is valid in this region. The other part is of the multiple tunneling path regime. The spectra are oscillating functions of $J$ in this region since $w \sim \cos (J \Omega / 2), \quad 0 \leqslant \Omega<2 \pi / 15$. Upon full monotonic variation of $\Omega$, the spectrum makes $\approx J / 30$ oscillations for both configurations. The spectrum of
TABLE XII. Spectra of $\mathcal{C}(\mathbf{Y}, 3)$; all eigenvalues are multiples of $w$.

\begin{tabular}{cc}
\hline \hline$J$ & Eigenvalues (degeneracies) \\
\hline 0 & $-\sqrt{5}(3),-2(4), 0(4), 1(5), \sqrt{5}(3), 3(1)$ \\
1 & $-(1+\sqrt{13}) / 2(5),-1(4),(3-\sqrt{5}) / 2(3)$, \\
& $(-1+\sqrt{13}) / 2(5),(3+\sqrt{5}) / 2(3)$ \\
2 & $-(3+\sqrt{5}) / 2(3),(1-\sqrt{13}) / 2(5),(3-\sqrt{5}) / 2(3)$, \\
& $1(4),(1+\sqrt{13}) / 2(5)$ \\
3 & $-3(1),-\sqrt{5}(3),-1(5), 0(4), 2(4), \sqrt{5}(3)$ \\
$1 / 2$ & $-(\sqrt{3}+\sqrt{7}) / 2(6), \sqrt{3}(1-\sqrt{5}) / 2(2)$, \\
& $(-\sqrt{3}+\sqrt{7}) / 2(6), \sqrt{3}(4), \sqrt{3}(1+\sqrt{5}) / 2(2)$ \\
$3 / 2$ & $-\sqrt{6}(4),-1(6), 1(6), \sqrt{6}(4)$ \\
$5 / 2$ & $-\sqrt{3}(1+\sqrt{5}) / 2(2),-\sqrt{3}(4),(\sqrt{3}-\sqrt{7}) / 2(6)$ \\
& $\sqrt{3}(-1+\sqrt{5}) / 2(2),(\sqrt{3}+\sqrt{7}) / 2(6)$ \\
\hline
\end{tabular}

$\mathcal{C}(\mathbf{Y}, 5)$ given in Table XI holds valid for both regimes. For the $\mathcal{C}(\mathbf{Y}, 3)$ configuration, in a range of parameters the proximity of the minima positions may be altered: each minimum position (vertex of the dodecahedron where some three faces intersect) should be geometrically connected not just to the three nearest neighbors but also to the six next-nearest neighbors.

\section{RANDOM ENERGY LEVELS}

For all configurations considered in the preceding section, the spectra were simple periodic functions of $J$, which was due to the fact that a rational number of flux quanta $[\Phi$ $=J \Omega(c)=2 \pi J P / Q]$ passes through each plaquette. This is not the case for more complex configurations such as $\mathcal{C}(G, 2), G=\mathbf{O}, \mathbf{Y}$. In Fig. 9 we present the spatial distribution of minima of the $\mathcal{C}(\mathbf{O}, 2)$ configuration. The segments connecting the minima are not real tunneling trajectories but rather guidelines. The tunneling paths may deviate strongly from the geodesics connecting corresponding minima both to the locations of the sixfold (centers of the cube faces) and eightfold (vertices of the cube) configurations' positions. The exact form of the paths depends on the CEF constants, e.g.,

TABLE XI. Spectra and low-temperature magnetic susceptibilities of $\mathcal{C}(\mathbf{Y}, 5)$; all eigenvalues are multiples of $w$ and all susceptibilities are multiples of $\left(g J \mu_{B}\right)^{2}\left[\beta=1 /\left(k_{B} T\right), c_{1}=\cos (\pi / 10)\right.$, and $c_{3}$ $=\cos (3 \pi / 10)]$.

\begin{tabular}{ccc}
\hline \hline$J$ & Eigenvalues (degeneracies) & Susceptibility \\
\hline 0 & $-\sqrt{5}(3),-1(5), \sqrt{5}(3), 5(1)$ & $(1+\sqrt{5}) /(6 w)$ \\
1 & $-\sqrt{5}(4),(\sqrt{5}-3) / 2(5),(5+\sqrt{5}) / 2(3)$ & $\beta / 9$ \\
2 & $-\sqrt{5}(4),(\sqrt{5}-5) / 2(3),(\sqrt{5}+3) / 2(5)$ & $\beta / 9$ \\
3 & $-(\sqrt{5}+3) / 2(5),(5-\sqrt{5}) / 2(3), \sqrt{5}(4)$ & $2 \beta / 9$ \\
4 & $-(5+\sqrt{5}) / 2(3),(3-\sqrt{5}) / 2(5), \sqrt{5}(4)$ & $\beta / 6$ \\
5 & $-5(1),-\sqrt{5}(3), 1(5), \sqrt{5}(3)$ & $(5+\sqrt{5}) /(30 w)$ \\
$\frac{1}{2}$ & $-2 c_{1}(6),(3-\sqrt{5}) c_{1}(4), 2 \sqrt{5} c_{1}(2)$ & $\beta / 5$ \\
$\frac{3}{2}$ & $-2 \sqrt{5} c_{3}(2),-2 c_{3}(6),(3+\sqrt{5}) c_{3}(4)$ & $\beta(5+\sqrt{5}) c_{1} /(15 w)$ \\
$\frac{5}{2}$ & $-\sqrt{5}(6), \sqrt{5}(6)$ & $\beta / 5$ \\
$\frac{7}{2}$ & $-2 \sqrt{5} c_{1}(2),(\sqrt{5}-3) c_{1}(4), 2 c_{1}(6)$ & $\beta / 9$ \\
$\frac{9}{2}$ & &
\end{tabular}


TABLE XIII. Low-temperature magnetic susceptibilities of $\mathcal{C}(\mathbf{Y}, 3)$ [a common factor of $\left(g J \mu_{B}\right)^{2}$ is omitted, $\left.\beta=1 /\left(k_{B} T\right)\right]$.

\begin{tabular}{lc}
\hline \hline$J$ & Susceptibility \\
\hline 0 & $(7 / 6+11 \sqrt{5} / 18) / w$ \\
1 & $\beta[12 \sqrt{5}+37+\sqrt{13}(3 \sqrt{5}+4)] / 468$ \\
2 & $\beta / 6$ \\
3 & $(\sqrt{5}+3) /(6 w)$ \\
$1 / 2$ & $\beta[10 \sqrt{5}+83+\sqrt{21}(5 \sqrt{5}-2)] / 630$ \\
$3 / 2$ & $\beta(4 \sqrt{5}+9) / 90$ \\
$5 / 2$ & $\beta / 9$ \\
\hline \hline
\end{tabular}

for the simplest Hamiltonian (6), where configuration $\mathcal{C}(\mathbf{O}, 2)$ is realized, it is a function of ratio $b / a$. Instead of studying nonuniversal tunneling trajectories, we introduce a parameter $\alpha(0<\alpha<2 \pi / 3)$ : the solid angle subtended by a squarelike contour. The solid angle subtended by a trianglelike circuit is $\pi / 2-3 \alpha / 4$. A knowledge of this parameter together with $w$ is sufficient to define the spectra of the 12-fold configuration. Since $\alpha$ may be an irrational multiple of $\pi$, the spectrum as a function of $J$ is not expected to be a finite set of values, but a fractal set. The spectra of the 12-fold configuration are given in Table XIV. The spectra undergo $J / 12$ oscillations upon a monotonic variation of $0<\alpha<2 \pi / 3$ for a given value of spin $J$.

The spectra found by Harter and Patterson [2] for this configuration are incorrect, since the spectra should depend on two parameters, e.g., on $w$ and $\alpha$, and not just on one (parameter $S$ in their work). However, the splitting schemes for $\mathcal{C}(\mathbf{O}, 2)$ found in [2] are correct (they are parameter independent).

Configuration $\mathcal{C}(\mathbf{Y}, 2)$ is even more complex than $\mathcal{C}(\mathbf{O}, 2)$. Its minima directions correspond to the midpoints of the icosahedron edges (see Fig. 10). The parameter $\alpha \quad(0 \leqslant \alpha$ $<\pi / 3$ ) here corresponds to the solid angle subtended by a pentagonlike contour. The spectra undergo $J / 30$ oscillations upon a monotonic variation of $0<\alpha<\pi / 3$. The spectra of the 30 -fold configuration for odd values of $J$ are given in Table XV.

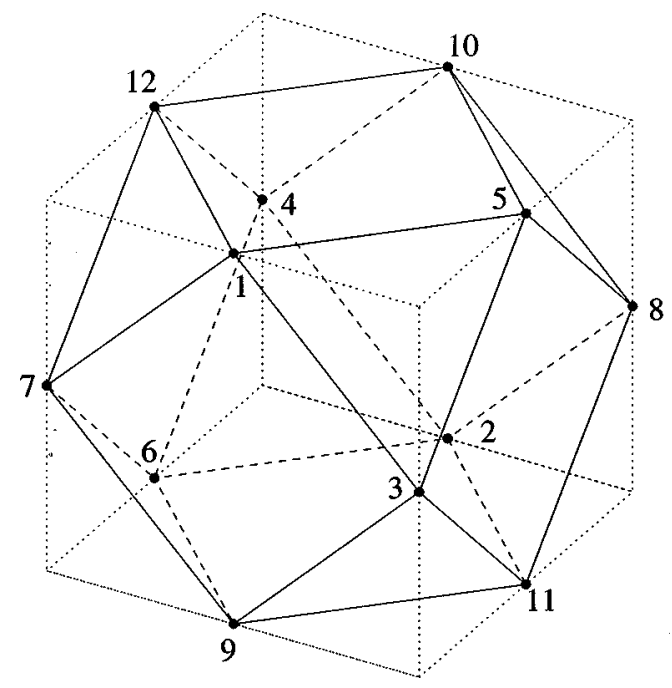

FIG. 9. Minima distribution of the 12-fold configurations of $\mathbf{O}$.
TABLE XIV. Spectra of $\mathcal{C}(\mathbf{O}, 2)$. All eigenvalues are multiples of $w ; x=J(\alpha+2 \pi) / 4$.

\begin{tabular}{lc}
\hline \hline \multicolumn{1}{c}{ Integer $J$} & Half integer $J$ \\
\hline Energy (degeneracy) & Energy (degeneracy) \\
\hline $4 \cos x(1)$ & $2(\cos x \pm \sqrt{2} \sin x)(2,2)$ \\
$-2 \cos (2)$ & $-\cos x \pm \sqrt{2+\cos ^{2} x}(4,4)$ \\
$2 \cos x(3)$ & \\
$-\cos x \pm \sqrt{8-7 \cos ^{2} x}(3,3)$ & \\
\hline \hline
\end{tabular}

The spectra described in this section have features of randomness. Indeed, the function $\{\alpha J\}$ (fractional part of $\alpha J$ ) with an irrational $\alpha$ is known as a generator of random numbers. Thus, the ratios of the transition frequencies for configurations $\mathcal{C}(G, 2), G=\mathbf{O}, \mathbf{Y}$ vary in an uncontrollable way when large $J$ changes by 1 . This behavior differs dramatically from that for other cubic and icosahedral configurations which display permanent ratios of the frequencies for a fixed configuration. Thus, the configurations $\mathcal{C}(G, 2), G=\mathbf{O}, \mathbf{Y}$ realize the chaotic spectra of deterministic systems. This situation is well known, e.g., for the hydrogen atom in a uniform magnetic field [16]. The peculiarity of our problem is that it displays chaos in a finite set of numbers (12 or 30) and that the chaotic behavior can be found analytically. Another special feature of our system is that stochasticity in it is combined with deterministic multiplicity distribution. For example, in the case of the $\mathcal{C}(\mathbf{O}, 2)$ configuration the 12 levels are divided into submultiplets given in Table I, independently of $\alpha$. However, their mutual arrangement is unpredictable.

For a two parametric Hamiltonian, e.g., Hamiltonian (6) for the octahedron group, the configurations $\mathcal{C}(G, 2), G$ $=\mathbf{O}, \mathbf{Y}$ correspond to the single tunneling regime. The multiple tunneling regime may occur if the invariants of higher orders are included.

In the presence of infinitely small magnetic field the ground state of configuration $\mathcal{C}(\mathbf{O}, 2)$ acquires either a finite magnetic moment or a finite susceptibility. We analyzed this problem for the field directed along one of the fourth-order axes and $w>0$. Then the finite magnetic moment $2 g J \mu_{B}|\sin x| /\left[2\left(8-7 \cos ^{2} x\right)\right]^{1 / 2}$ is acquired at $\cos x>-1 / 2$ $[x=J(\alpha+2 \pi) / 4]$, otherwise the finite magnetic susceptibility $\chi=-\left(g J \mu_{B}\right)^{2} /(3 w \cos x)$ occurs for integer $J$. For half

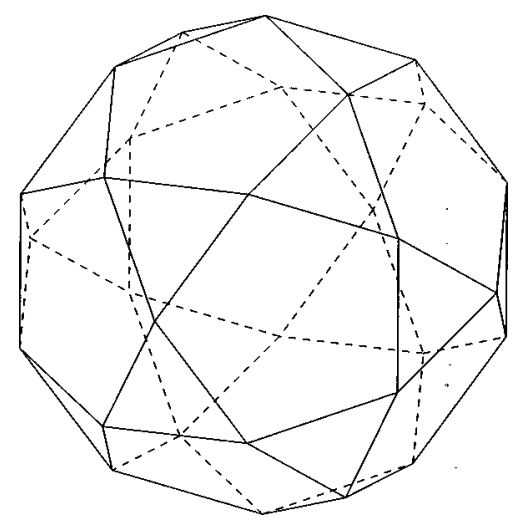

FIG. 10. Minima distribution of the 30-fold configurations of $\mathbf{Y}$. 
TABLE XV. Spectra of $\mathcal{C}(\mathbf{Y}, 2)$ for odd values of $J$. All eigenvalues are multiples of $w, x=\cos [J(\alpha+3 \pi) / 5]$.

\begin{tabular}{ll}
\hline \hline Energy (degeneracy) \\
\hline $1+2 x \quad(4,4)$ \\
$-x \pm \sqrt{4-3 x^{2}}(5,5)$ \\
$(1+\sqrt{5})\left[-x \pm \sqrt{4+(5-4 \sqrt{5}) x^{2}}\right] / 2 \quad(3,3)$ \\
$(1-\sqrt{5})\left[-x \pm \sqrt{4+(5+4 \sqrt{5}) x^{2}}\right] / 2 \quad(3,3)$ \\
\hline
\end{tabular}

integer $J$, the magnetic moment $g J \mu_{B} / 3$ is acquired at $\cos x$ $<\cos (3 \pi / 8)$, otherwise this value of the moment is multiplied by a factor

$$
\left(\frac{c^{2}+5+3 c \sqrt{2+c^{2}}+2 \sqrt{2}|\sin (x)|\left(3 c+\sqrt{2+c^{2}}\right)}{2\left(2+c^{2}\right)}\right)^{1 / 2},
$$

where $c=\cos (x)$. Note the random character of these values.

\section{NUMERICAL ANALYSIS. THE CASE OF THE CUBIC CEF}

The main obstacle to a reliable numerical analysis of the problem is the fact that nobody knows what the Hamiltonian looks like. The case of the rare-earth ions with large total angular momenta interacting with the CEF represents an exception. Only the orbital part $\mathbf{L}$ of the total angular momentum of a single magnetic electron interacts with the crystalline field. All terms, in the expansion of the crystalline potential with the degree larger than $2 l$, where $l$ is the orbital quantum number of the single magnetic electron, vanish [17], thus simplifying the analysis. For the $4 f$-group electrons with $l=3$, this gives the highest non-vanishing terms of the sixth order. Considering a CEF of a particular symmetry group brings further simplification, e.g., in the cubic CEF, there are only two independent invariants of the sixth order and one of the fourth order. The two of the sixth order are combined in one invariant [see Eq. (6)] for a real interaction which is the Coulomb interaction between the charge carriers.

It has been shown in Sec. II that Hamiltonian (6) has configurations $\mathcal{C}(\mathbf{O}, 4), \mathcal{C}(\mathbf{O}, 3)$, and $\mathcal{C}(\mathbf{O}, 2)$ as sets of its classical extrema. In this meaning it is rather general. Therefore, we apply numerical analysis to this Hamiltonian in a wide range of $J$ 's. This means that we numerically diagonalize the $(2 J+1) \times(2 J+1)$ matrix for a one-parametric set of Hamiltonians (29). The choice of this Hamiltonian is partly justified by the above consideration. Our purpose is to find numerically what $J$ can be considered as large, i.e., starting from what $J$ our theory gives a satisfactory description. The second important problem is the crossover behavior of the spectrum near configuration boundaries described in Sec. II.

Numerical studies of the crystal field effects on angular momenta were performed in the early 1960s by Lea, Leask, and Wolf [18]. These authors studied a cubic crystal field Hamiltonian similar to Eq. (6). Their main interest was how the angular momentum degeneracy of $f$ electrons is lifted. For this purpose it was enough to consider values of $J$ spanned from 3 to 8 . Numerical studies for high values of $J$ (up to 100) were carried out by Fox, Galbraith, Krohn, and

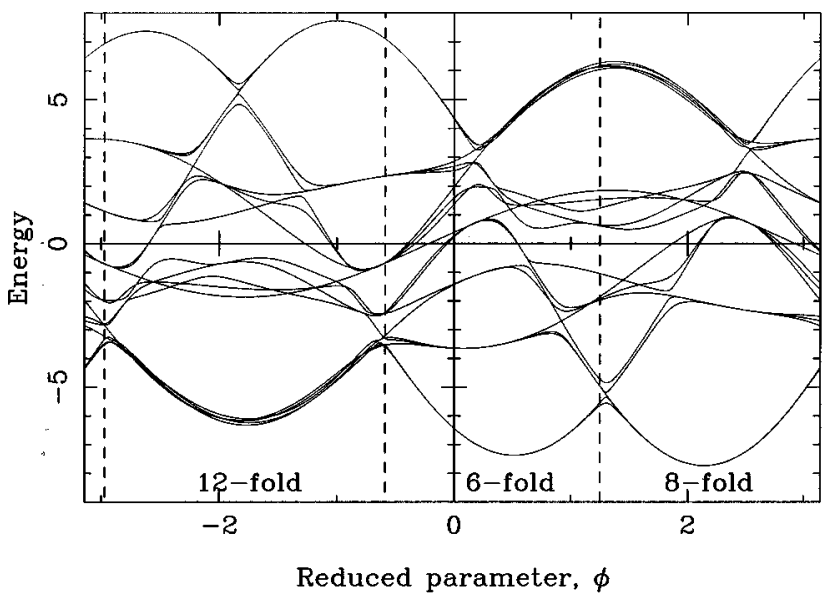

FIG. 11. Spectrum of Hamiltonian (29); $J=23$.

Louck [19]. However, this group used a Hamiltonian similar to Eq. (3) with the fourth-order term only. Avoiding this limitation, we numerically study the Hamiltonian consisting of terms of the fourth and sixth order for an arbitrary value of $J$. However, we use a different parametrization than that used in [18] for the same Hamiltonian:

$$
H_{2}^{\mathbf{O}}=-\frac{\cos (\phi) O_{4}^{0}}{[J(J+1)]^{2}}-\frac{5}{14} \frac{\sin (\phi) O_{6}^{0}}{[J(J+1)]^{3}},
$$

where $O_{4}^{0}$ and $O_{6}^{0}$ are Stevens's operator equivalents [20,17], and $\phi$ is a parameter taking values in the interval $[-\pi, \pi]$. Our parametrization corresponds to a unit circle on the phase diagram of Hamiltonian (6) (see Fig. 3): $a=\cos (\phi)$, $b=\sin (\phi)$, whereas that chosen in [18] corresponds to the square: $a=x, 14 b / 5= \pm(1-|x|) ;-1 \leqslant x \leqslant 1$. The coefficient of $5 / 14$ reflects the difference between our invariant of the sixth order in Eq. (6) and the commonly used Stevens operator equivalent $O_{6}^{0}$.

For relatively small values of spins $2 J+1 \sim N$, where $N$ is the number of extrema of the CEF, the quasiclassical description fails and the spectrum of Hamiltonian (29) does not follow the predicted dependence. However, for $J \approx 10$, one can observe distinct regions of $\phi$ with high density of level crossing (these regions are distinctly seen in [18] for $J \geqslant 6$ ). Upon an increase of $J$ these regions narrow down, giving the points separating the sixfold, eightfold, and 12-fold configurations. Further increase of $J$ leads to a "bunching" of low energetic levels into the predicted groups (multiplets) of six, eight, or 12 .

Not only the numbers of the levels in the multiplets, but also the ratios of the spacings between the levels inside the multiplets, the oscillations of the spectra in the regime of the multiple tunneling path, and the tunneling amplitude in the regime of a single tunneling path obey the predictions of our theory.

For a demonstration we have chosen a set of close valued $J$ 's: $J=23,47 / 2$, and 24. Figures 11, 12, and 13 are graphs of the spectra of Hamiltonian (29) for these values of $J$. The vertical dashed lines are the classical boundaries between the different configuration [see the diagram of Hamiltonian (6), Fig. 3]. A small deviation of the dashed line separating the sixfold and eightfold configurations $\left[\phi_{6-8}=\arctan (3)\right]$ to- 


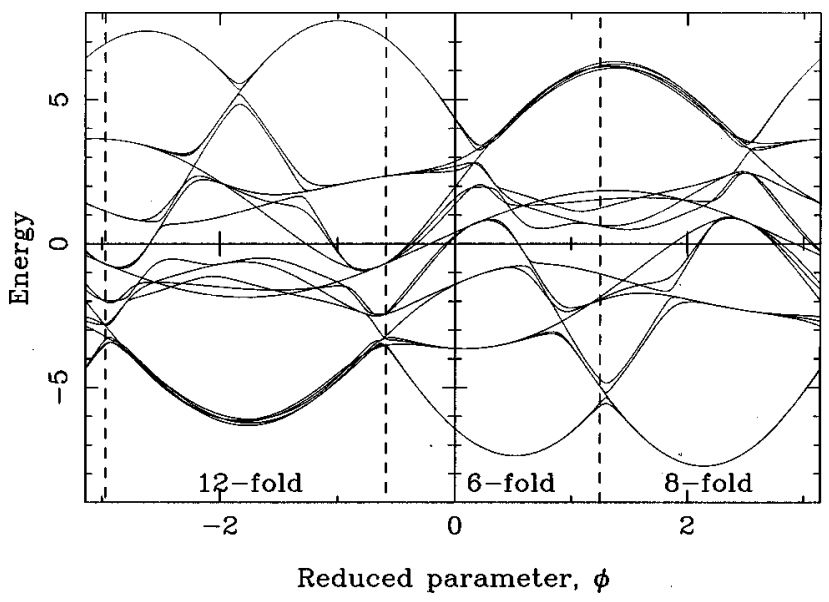

FIG. 12. Spectrum of Hamiltonian (29); $J=47 / 2$.

wards the sixfold one is due to the fact that, at $\phi=\phi_{6-8}$, the depth of the CEF potential in the minimum locations of the sixfold configuration is equal to that of the eightfold configuration. However, the intersection of the levels occurs when the ground-state energies coincide. See Appendix D for details on this subject.

From the pictures one can clearly see the "bunching" of the highest- and lowest-energy levels into the predicted multiplets of six, eight, and 12. The excited multiplets have the same structure which fails only in the vicinity of the boundaries between the configurations. The structure of the spectra given in Figs. 11, 12, and 13 looks quite similar at this level of "magnification." To see the subtle details predicted in previous sections we should "zoom in" the pictures, "focusing" on the ground multiplet.

First, we shift the "center of mass" of the ground multiplet to zero (we are not interested in finding the single-well localization energy). Secondly, we rescale the shifted levels, so that a "visual" comparison of the spacings between the levels can be done at different values of the reduced parameter $\phi$. The rescaling is necessary due to a large variation of $w \propto \exp [-J c(\phi)]$. The calculations of the tunneling amplitude for the $\mathcal{C}(\mathbf{O}, 4)$ configuration of Hamiltonian (6) (see Fig. 2) predict a variation of $w$ of order $10^{6}$ for $J \approx 24$. The results of this program are shown in Figs. 14, 15, and 16 for $J=23$, $47 / 2$, and 24, respectively. The vertical dashed lines (the

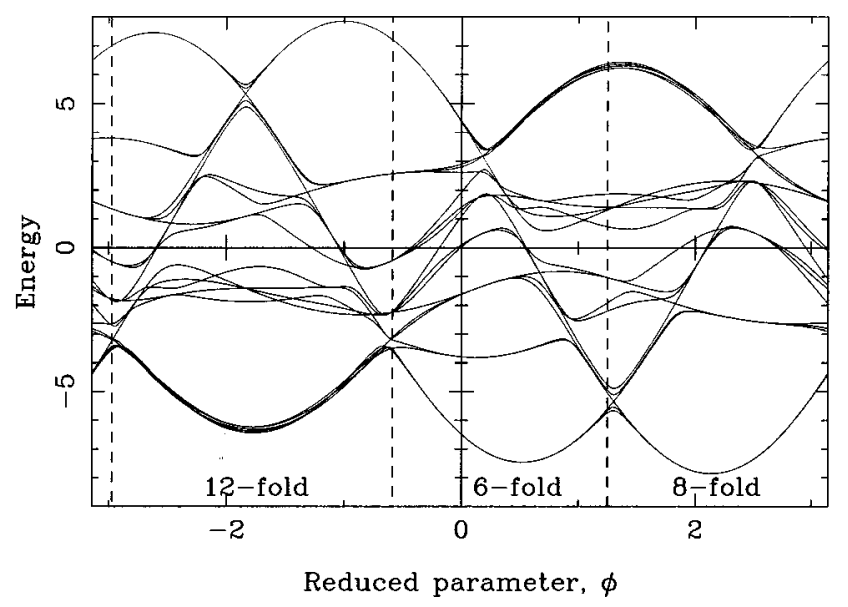

FIG. 13. Spectrum of Hamiltonian (29); $J=24$.

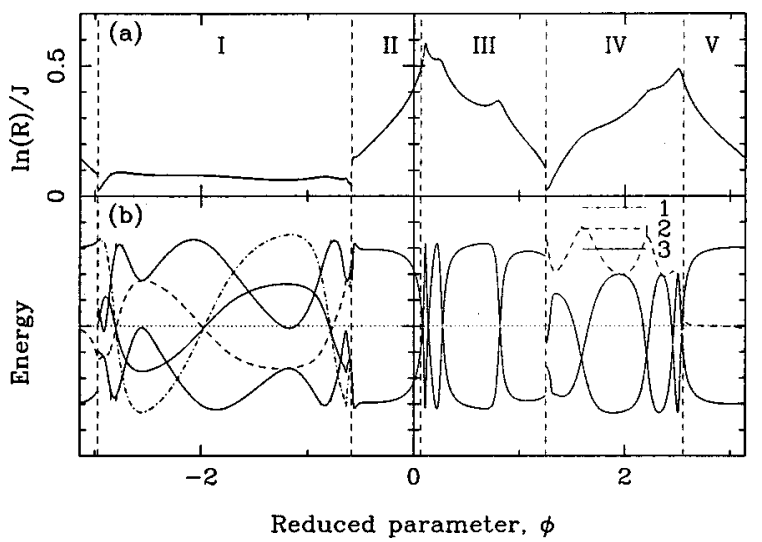

FIG. 14. (a) Graph of $\ln (R) / J$, where $R$ is the rescaling factor applied to the ground multiplet; (b) rescaled ground-state multiplet of Hamiltonian (29) (the legend shows the degeneracies of the levels); $J=23$.

quasiclassical boundaries, see Fig. 3) separate not only the regions of different configuration numbers but also the regions of the single and multiple tunneling path regimes. The regions are enumerated by Roman numerals: $\mathrm{I}-\mathcal{C}(\mathbf{O}, 2)$ (single tunneling path regime only), II $-\mathcal{C}(\mathbf{O}, 4)$ single, III- $\mathcal{C}(\mathbf{O}, 4)$ multiple, IV $-\mathcal{C}(\mathbf{O}, 3)$ multiple, and $\mathrm{V}-\mathcal{C}(\mathbf{O}, 3)$ single regimes, respectively. Part (a) of each picture represents the plot of the rescaling factor which is proportional to $c(\phi)$; part (b) is the rescaled spectrum.

All predictions of Secs. II, V, and VI (the orderings of the levels, the ratios of the level spacings, the oscillations of the spectra in some regions, the numbers of the oscillations, and the dependence of the scaling parameter $R$ ) find confirmation here. The oscillations are not of the periodic form due to nontrivial (but monotonic) dependencies $\alpha=\alpha(\phi)$ and $\Omega$ $=\Omega(\phi)$.

A more precise value of $c(\phi)$ can be easily obtained from the single tunneling path part of the spectrum of the sixfold configuration. Figure 17 compares the quasiclassical result found in Sec. II with the numerical calculations for $J=24$ and 48. The plot is $-\ln \left[\left(E_{1}-E_{0}\right) / 4\right] / J$ vs $u=\tan (\phi)$, where $E_{0}$ and $E_{1}$ are the energies of the ground and first excited states, respectively. The difference $E_{1}-E_{0}$ is $4 w$ according

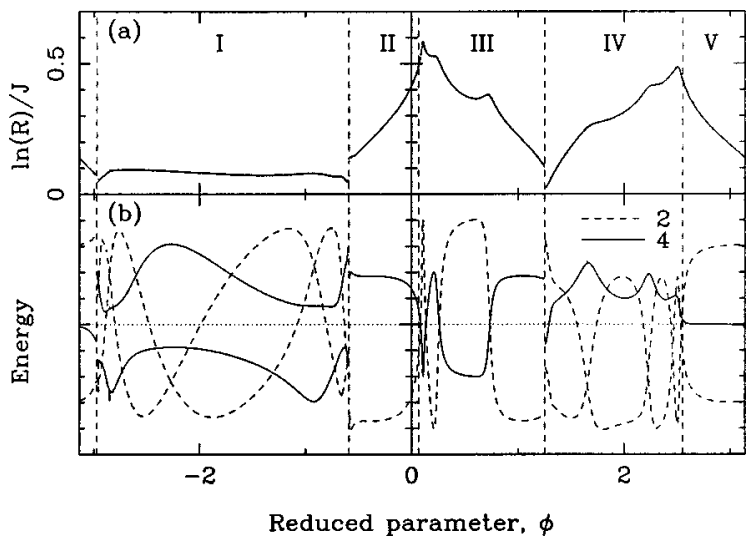

FIG. 15. (a) Graph of $\ln (R) / J$, where $R$ is the rescaling factor applied to the ground multiplet; (b) rescaled ground-state multiplet of Hamiltonian (29) (the legend shows the degeneracies of the levels); $J=47 / 2$. 


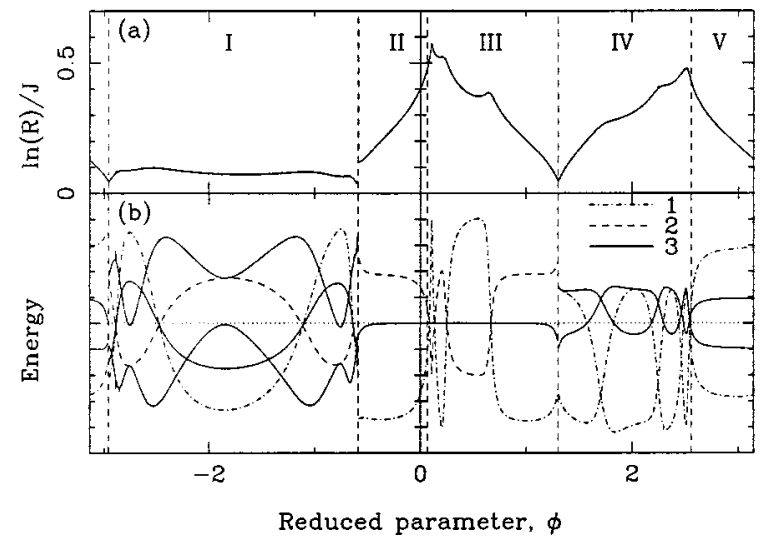

FIG. 16. (a) Graph of $\ln (R) / J$, where $R$ is the rescaling factor applied to the ground multiplet; (b) rescaled ground-state multiplet of Hamiltonian (29) (the legend shows the degeneracies of the levels); $J=24$.

to predictions of Sec. V. A small discrepancy is due to the coefficient of the exponential $f(u)(w=f(u) \exp [-J c(u)])$, whose contribution decreases $\propto 1 / J$. From these data, we can estimate that the values of the coefficient $f(u)$ are in a range $0.1-3.0$.

All these facts strongly emphasize the validity of the developed quasiclassical description of the large spins from the theoretical point of view. Now questions arise: What is a possible experimental realization? What are the limitations of the theory when applied to the real systems? We will discuss these questions in the next section.

\section{EXPERIMENTAL REALIZATION}

\section{A. Feasible experimental systems}

The experimental observation of the predicted effects can be done on any system with large values of the angular momentum such as rare-earth-metal ions, magnetic clusters, or nuclei. The main question is whether the value of $J$ is large enough for a given configuration of the external field.

For a configuration with small number of minima (twofold and fourfold configuration), $J \approx 8$ satisfies the quasiclassical requirement. Such values of $J$ are available, e.g., in rare-earth-metal ions: $\mathrm{Dy}^{3+}, \mathrm{Ho}^{3+}$, or $\mathrm{Er}^{3+}$. An example of compounds with the tetragonal symmetry, where the fourfold configuration is realized, is $\mathrm{RENi}_{2} \mathrm{~B}_{2} \mathrm{C}$, $\mathrm{RE}$ stands for a rareearth magnetic element. To suppress the influence of the in-

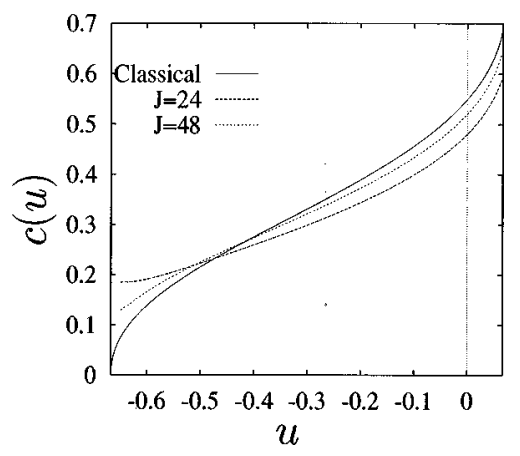

FIG. 17. Comparison of the quasiclassical and numerical tunneling amplitude exponent, $u=\tan (\phi)$. teraction between the magnetic moments the magnetic ions should be diluted with similar but nonmagnetic ones such as $\mathrm{La}^{3+}, \mathrm{Lu}^{3+}$, or $\mathrm{Y}^{3+}$. The CEF effects for this family of compounds were studied in single crystals of $\mathrm{Lu}_{1-x} \mathrm{Ho}_{x} \mathrm{Ni}_{2} \mathrm{~B}_{2} \mathrm{C}$ by Cho et al. [21]. The calculated CEF level scheme given there shows that the ground-state quadruplet is well separated from other excited states and corresponds to the multiplet of $\mathcal{C}\left(\mathbf{D}_{4}, 2\right)$ configuration with $J=0$ $(\bmod 2)$ and $w \approx 2 \mathrm{~K}$.

Another family of rare-earth compounds, RESb, offers the cubic environment. However, it is questionable whether the quasiclassical requirement is satisfied since even for the highest values of the angular moment $\left(J=8\right.$ for $\left.\mathrm{Ho}^{3+}\right)$ the multiplicity $2 J+1=17$ is not so large comparatively to the lowest dimension of the cubic configurations $N=6$. The numerical calculations performed in the preceding section indicate that only for $J \geqslant 12$ are there regions of parameter $\phi$ where the sixfold and eightfold configurations are well defined. To obtain the 12-fold configuration, in the framework of Hamiltonian (29), the value of $J$ should be increased to about 24.

Magnetic clusters and molecules offer systems with very large total spins and a variety of symmetries. Theoretical calculations [22] indicate that clusters of 13 atoms of transition metals such as Fe, Pd, and Rh may have cubic symmetry and total magnetic moment of the order of $\mu_{B}$ per atom. Gadolinium clusters $\operatorname{Gd}_{n}(n=11-92)$ [23] exhibit large magnetic moments of $(0.5-3.0) \mu_{B}$ per atom (which is below the bulk value of $7.55 \mu_{B}$ but still offers a large value of the total cluster spin) with behaviors ranging from tight locking to the lattice by crystal anisotropy to superparamagnetism (almost free moment).

Large spins were also observed in artificially grown magnetic dots used for observation of the magnetic tunneling [24]. So far, these systems belonged to the lowest symmetry class. It is rather tempting to create environment of higher symmetry and to use smaller magnetic dots like the ones used by Schuller and co-workers [25] to observe the effects predicted by our theory.

\section{B. Practicable experiments. Magnetic measurements}

The experimental consequences of the difference among the configurations and the spin values can be observed with many experiments. To name a few these are measurements of the spin magnetic moment and magnetic susceptibility, relaxation of the magnetization, electron paramagnetic resonance (EPR), and nuclear magnetic resonance (NMR).

First we discuss measurements of the magnetic susceptibility. The magnetic susceptibility follows the Curie law for temperatures higher than the characteristic splitting of the ground-state multiplet $T k_{B}>w$ (see Appendix C). Thus, it is $\left(g \mu_{B} J\right)^{2} /\left(k_{B} T\right)$ for one-dimensional configurations, i.e., $\mathcal{C}\left(\mathbf{D}_{N}, N\right), \quad N=2,4,6,\left(g \mu_{B} J\right)^{2} /\left(2 k_{B} T\right)$ for twodimensional ones, i.e., $\mathcal{C}\left(\mathbf{D}_{N}, 2\right), \quad N=4,6$, and $\left(g \mu_{B} J\right)^{2} /\left(3 k_{B} T\right)$ for the three-dimensional ones, i.e., for the rest of the configurations considered in this work. For temperatures lower than the characteristic splitting $T k_{B}<w$, the Curie dependence is no longer universal. The nonmagnetic classes, that is, those without magnetic moment in the ground state, have their magnetic susceptibility saturated to 


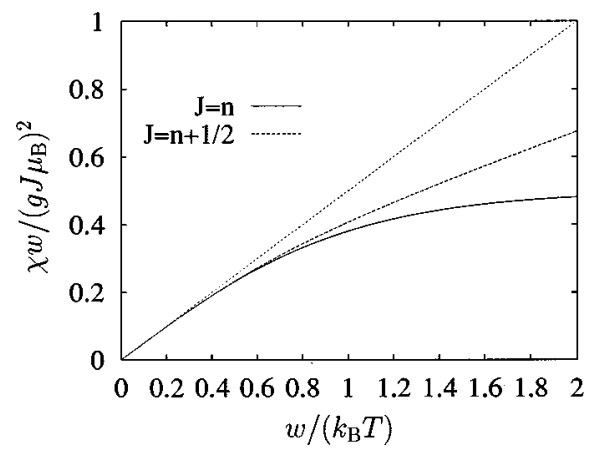

FIG. 18. Magnetic susceptibility vs inverse temperature. $\mathcal{C}\left(\mathbf{D}_{4}, 2\right)$ configuration. The dotted line is the high-temperature asymptote.

csome constant at $T \rightarrow 0$, whereas the magnetic ones (with a nonzero magnetization in the ground state) still obey the Curie-like behavior. Both the saturation values and the slopes of the Curie-like curves depend upon the configuration of the symmetry group as well as upon the equivalence class of the spin. For example, the twofold configurations $\mathcal{C}\left(\mathbf{D}_{N}, N\right)$ are nonmagnetic for $J \cong N / 2, N=2,4,6$; the saturation values of the magnetic susceptibility are $\chi(T)$ $=\left(g \mu_{B} J\right)^{2} /|E(0)|$, where $E(0)$ are the corresponding eigenvalues for zero magnetic field [see Eqs. (22)]. The magnetic classes of these configurations, i.e., the ones with $J \cong N / 2$, have the same Curie-like dependence: $\chi(T)$ $=\left(g \mu_{B} J\right)^{2} /\left(k_{B} T\right)$.

In the case of the $\mathcal{C}\left(\mathbf{D}_{N}, 2\right)$ configurations, $N=4,6$, the integer spin classes are non-magnetic and the half-integer ones are magnetic. The low-temperature magnetic susceptibilities of these configurations can be found in the last column of Tables VI, VII for the $\mathcal{C}\left(\mathbf{D}_{4}, 2\right)$ and $\mathcal{C}\left(\mathbf{D}_{6}, 2\right)$ configurations, respectively. A detailed temperature dependence of the magnetic susceptibility is shown in Fig. 18 for the $\mathcal{C}\left(\mathbf{D}_{4}, 2\right)$ configuration.

The division into the classes of equivalence is more subtle for the high-order symmetry groups. Tables VIII, IX, XI, and XIII collect the low-temperature susceptibilities for the $\mathcal{C}(\mathbf{O}, 4), \mathcal{C}(\mathbf{O}, 3), \mathcal{C}(\mathbf{Y}, 5)$, and $\mathcal{C}(\mathbf{Y}, 3)$ configurations, respectively. Figures 19 and 20 show the details of the transition from the Curie high-temperature regime to the lowtemperature one for the $\mathcal{C}(\mathbf{O}, 4)$ and $\mathcal{C}(\mathbf{O}, 3)$ configurations, respectively.

For magnetic measurements it is important that the system is in thermal equilibrium and the range of temperature $T k_{B}$

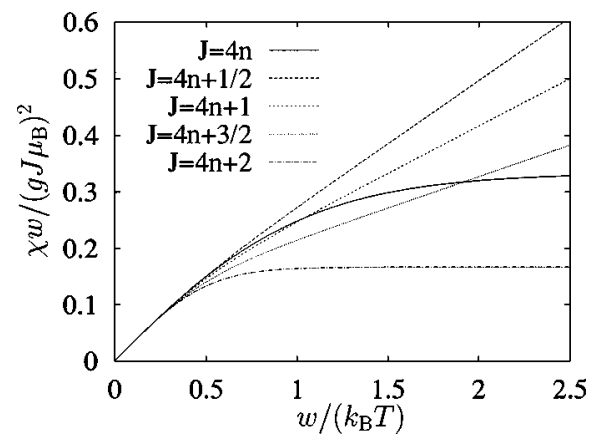

FIG. 19. Magnetic susceptibility vs inverse temperature. $\mathcal{C}(\mathbf{O}, 4)$ configuration.

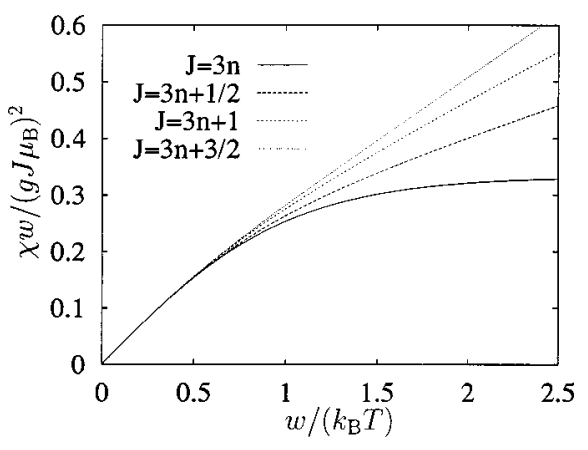

FIG. 20. Magnetic susceptibility vs inverse temperature. $\mathcal{C}(\mathbf{O}, 3)$ configuration.

$<w$ is accessible. This requirement means that $w \sim \varepsilon_{0} e^{-c J}$ is not too small. On the other hand, $J$ must be not less than $\sim N / 2$ to guarantee the validity of quasiclassical approximation. At a fixed lower limit for experimentally accessible temperature $T_{l}$ inequality $N<J<\ln \left[\varepsilon_{0} /\left(k_{B} T\right)\right] / c$ must be satisfied. For rare-earth ions $\varepsilon_{0}$ is the atomic scale of energy and $N=6,8$. It gives $T_{l}<\varepsilon_{0} e^{-4} \sim 100 \mathrm{~K}$, which is easily satisfied. For $\mathrm{La}_{1-x} \mathrm{Ho}_{x} \mathrm{Ni}_{2} \mathrm{~B}_{2} \mathrm{C}$ the numerically estimated $w$ is about $2 \mathrm{~K}$ [21]; preliminary experimental results by Naugle and co-workers give $w \approx 1 \mathrm{~K}[26]$.

$\mathrm{Gd}^{3+}$ ion has zero orbital momentum, its anisotropy is caused by the relativistic spin-other-orbit interaction, and the corresponding $\varepsilon_{0}$ is about $10^{-4}$ time less than the atomic scale $\left(\varepsilon_{0} \sim 1-10 \mathrm{~K}\right)$. The total spin of the $\mathrm{Gd}^{3+}$ ion $S$ $=7 / 2$ is not too large, but may be enough in the case of the tetragonal symmetry. The estimated value $T_{l}$ is between 0.1 and $1 \mathrm{~K}$.

The anisotropy of a ferromagnetic cluster is induced mainly by its boundaries. The anisotropy energy has the same magnitude $\sim 1-10 \mathrm{~K}$ per a site near the boundary. For the cluster as a whole this value must be multiplied by the number of atoms on one of the faces of the cluster $(\mathcal{F})$ which depends on the cluster geometry. An estimate can be attained for the series of magic atom-number clusters [22]: $M_{\mathcal{N}}, \mathcal{N}$ $=13,55,147, \ldots$. These clusters are obtained by surrounding a core atom progressively with additional shells of atoms: $S_{k}=10 k^{2}+2, k=1,2,3, \ldots$. This procedure can be done for icosahedral, decahedral, and cuboctahedral packings, which have 20,15 , and 12 faces, respectively. For $\mathcal{N}$ $=55$ we find $\mathcal{F} \approx 42 / 16$ and $\varepsilon_{0} \approx 3-26 \mathrm{~K}$. On the other hand, $J \propto \zeta \mathcal{N}$. In the Gd cluster $\zeta \sim 0.5$ and for $\mathcal{N}=55$ we find $J \sim 27$. It is sufficiently large. The value of $w \sim \varepsilon_{0} e^{-c J}$ with $c \approx 0.3$ is between 0.001 and $0.01 \mathrm{~K}$. For $\mathcal{N}=13, w$ ranges between 0.1 and $1 \mathrm{~K}$.

\section{Spectral analysis}

The most straightforward experimental approach is the spectral analysis. The main difficulty with this method is that the scale of the splitting is very different for different systems and values of $J$. Nevertheless, we can expect that the spectral frequencies are either in the submillimeter or in the uhf range. Apart from the direct attenuation measurements, it is possible to apply EPR technique. It measures the splitting in magnetic field, i.e., magnetic moment in some state. The advantage of this method is that it does not require too low 
temperatures. Certainly, its sensitivity drops with the growth of temperature, but not too fast.

\section{Oscillations of magnetization}

Let us consider many identical large spins placed in an external magnetic field along one of the easy directions $(k)$, sufficiently large to polarize them almost to saturation. If the field is switched off abruptly, each spin remains in the same state $|k\rangle$. Since $|k\rangle$ is not a stationary state, it will vary in time according to the Schrödinger picture:

$$
|k, t\rangle=\sum_{j \alpha}|j \alpha\rangle\langle j \alpha \mid k\rangle e^{-i E_{j} t / \hbar}
$$

Here $j$ labels sublevels of one $N$-plet and $\alpha$ labels states of the $j$ th sublevel. It leads to oscillation of the magnetic moment along the $k$ direction in time:

$$
\begin{aligned}
M(t)= & g \mu_{B} J \sum_{k^{\prime}} \cos \gamma_{k k^{\prime}} \sum_{j \alpha, j^{\prime} \alpha^{\prime}}\langle j \alpha \mid k\rangle \\
& \times\left\langle j^{\prime} \alpha^{\prime} \mid k\right\rangle^{*}\left\langle j \alpha \mid k^{\prime}\right\rangle^{*}\left\langle j^{\prime} \alpha^{\prime} \mid k^{\prime}\right\rangle e^{-i \omega_{j j^{\prime}} t},
\end{aligned}
$$

where $\gamma_{k k^{\prime}}$ is the angle between the directions of classical angular momentum in the extrema $k$ and $k^{\prime}$, and $\omega_{j j^{\prime}}=\left(E_{j}\right.$ $\left.-E_{j^{\prime}}\right) / \hbar$ is the transition frequency. All spins had the same initial state $|k\rangle$ at the moment when the field was switched off, therefore, their magnetic moment will rotate coherently, creating the macroscopic rotating magnetization. Obviously, the rotation energy will dissipate. Let us estimate the attenuation time $\tau$. We assume that the spins are embedded in an insulator. Then only phonons lead to dissipation. The spinphonon interaction energy can be written as follows:

$$
\mathcal{H}_{s-\mathrm{ph}}=\lambda u_{\alpha \beta} J_{\alpha} J_{\beta},
$$

where $u_{\alpha \beta}$ is the deformation tensor. The value of the coupling constant can be estimated as $\lambda \sim \Delta / J^{2}$, where $\Delta$ is the energy difference of two oscillatory levels localized near one minimum of the potential $f(\mathbf{J})$. A routine calculation leads to an estimate of the oscillation lifetime $\tau$ :

$$
\tau \sim \frac{\hbar \rho s^{5}}{\Delta^{2} \omega^{3}},
$$

where $\rho$ is the mass density of the matrix and $s$ is the sound velocity. For typical values $\rho=10 \mathrm{~g} \mathrm{~cm}^{-3}, \Delta=10 \mathrm{~K}, \omega$ $=w / \hbar=10^{10} \mathrm{~s}^{-1}$, and $s=10^{5} \mathrm{~cm} \mathrm{~s}^{-1}$, we find $\tau$ $\sim 10^{-1}$ s. The magnetic field must be switched off for a shorter time interval. It seems feasible. For $\mathrm{Gd}$ we estimated both $\Delta$ and $w$ by a factor of 10 smaller than the values we used for the above estimate. It gives the attenuation time $\tau$ in the range of a few hours.

In our estimate we assumed that the temperature $T$ is less than or of the order of $w$. If it is much larger, the value of $\tau$, Eq. (33), must be multiplied by a small factor $\hbar \omega /\left(k_{B} T\right)$. At a temperature $1 \mathrm{~K}$ with $\hbar \omega \sim 0.01$ it changes $\tau$ from a few hours to a minute, but still leaves this time long. Thus, the requirement for temperature is not too restrictive.

Nevertheless, the observation of the macroscopic oscillations of magnetization may be obstructed because of inho- mogeneous line broadening caused by the dipolar interaction [27]. Indeed, the random shift of the frequency due to the dipolar interaction is of the order

$$
\delta \omega \sim \frac{g^{2} \mu_{B}^{2} J^{2}}{\hbar R^{3}}=\frac{g^{2} \mu_{B}^{2} J^{2} n x}{\hbar},
$$

where $R$ is the average distance between large spins, $x$ is their concentration per site, $n$ is the density of the matrix sites. For $g=2, J=3.5$, and $n=10^{22} \mathrm{~cm}^{-3}$ we find $\delta \omega$ $\approx 1.8 \times 10^{10} x \mathrm{~s}^{-1}$. For $x=0.001$ it is three orders of magnitude less than $\omega \sim 10^{10} \mathrm{~s}^{-1}$, but it destroys the coherence for the time interval $2 \pi(\delta \omega)^{-1} \sim 10^{-7} \mathrm{~s}$. What can be observed after this interval of time is the noise in a rather narrow spectral range $\delta \omega$ given by Eq. (34) near the frequency $\omega$. The noise attenuates during the interval $\tau$ [Eq. (33)] after the pulse of magnetic field. Repeating the pulse of magnetic field periodically with the period $t<\tau$, one can maintain a permanent average level of the noise. Also, one can use this narrow-line noise to generate coherent oscillations in a resonator.

\section{E. Rotation-vibrational spectra of molecules}

Harter and Patterson applied their theory [2] to experimentally observed (laser absorption spectra) splitting of rotational levels in a molecule $\left(\mathrm{SF}_{6}\right)$ with the cubic symmetry [28]. In the leading approximation this molecule can be considered as a spherical top. Due to weak rotation-vibration interaction the first cubic invariant $J_{x}^{4}+J_{y}^{4}+J_{z}^{4}$ occurs in the perturbed Hamiltonian. The splitting of the $2 J+1$ rotational states into several clusters has been found in spectroscopic experiments [28] for $J=17,18$, and 19. The outer clusters can be treated as 6- and 8-plets. Unfortunately the tiny tunneling splitting of these clusters is too small to be resolved spectroscopically.

\section{CONCLUSION}

We have shown that large spins (total orbital momenta) $J$ placed into external fields of high symmetry group $G$ display unusual behavior of low-lying and high-lying parts of spectra and magnetic susceptibility. These parts of spectra are represented by multiplets containing $N(G, p)$ states each, where $N(G, p)$ is the doubled number of $p$-fold axes. Each multiplet is split into sublevels with multiplicities chosen from dimensionalities of the irreducible representations of the point group $G$ and determined by $G, J$, and $p$. The distances between sublevels in the multiplet are proportional to $\exp (-c J)$, whereas the distances between multiplets are proportional to $1 / J$. The multiplicities at a fixed $G$ and $p$ are periodic functions of $J$ with the period $p$. The relative distances between levels are also periodic functions on $J$, but their period is equal to half of the number of the equivalent plaquettes formed by the tunneling trajectories and covering the unit sphere. Interesting exclusions are the configurations of the octahedron and icosahedron groups with $p=2$. In these cases the mutual arrangement of the levels is stochastic, though the multiplicities remain fully deterministic.

In all situations considered with the exception of the tetrahedral and hexagonal symmetry with in-plane easy direc- 
tions, the change of large spin $J$ by 1 leads to a drastic change in the spectrum and thermodynamic properties. We demonstrated that at such a change the magnetic susceptibility can either change its behavior from Curie law to saturation or change the coefficient in Curie law.

Rather special phenomena appear near hypersurfaces in the space of the Hamiltonians which separate regions with different configurations of the extrema of the potential, i.e., regions with different $N=N_{1}, N_{2}$. Directly on these hypersurfaces the number of equivalent extrema is equal to $N_{1}$ $+N_{2}$. Thus, in a narrow vicinity of the hypersurface there appears a new "class of universality," new set of sublevels with new multiplicities. Moreover, we expect a kind of "turbulent' behavior of levels near these hypersurfaces.

Given the classical Hamiltonian $\mathcal{H}(\mathbf{J})$, one can indicate a value $J_{c}(\mathcal{H})$, starting from which the multiplicities are correctly determined by our theory. Though this value is model dependent, our numerical calculations show that $J_{c}$ $\approx N(G, p)$.

All conclusions of the theory were checked numerically for a model Hamiltonian of the cubic symmetry containing two invariants (two free parameters) up to $J=60$. The agreement for the relative distances between the levels is very good starting from $J \approx 20$. Multiplicities are well determined by our theory starting from $J \approx 12$ for the sixfold and eightfold configurations and from $J \approx 16$ for the 12 -fold configuration.

We proposed three classes of experimental systems which can display the predicted effects. One of them is represented by alloys with participation of two lanthanides or actinides, $R$ and $R^{\prime}$, so that $R$ has zero orbital momentum and its concentration is close to 1 , whereas the element $R^{\prime}$ has large $J$ and its concentration is very small. In this way the configuration of large spin in a symmetric environment is realized. Typical representatives are $\mathrm{La}_{1-x} \mathrm{Ho}_{x} \mathrm{Ni}_{2} \mathrm{~B}_{2} \mathrm{C}$ (tetragonal environment) or $\mathrm{Lu}_{1-x} \mathrm{Dy}_{x} \mathrm{Sb}$ (cubic environment).

The second class of systems is metallic or metallo-organic clusters made from ferromagnetic metals. For such clusters symmetry can be not only octahedral, but also icosahedral, as it is for the cluster $\mathrm{Fe}_{13}$. The clusters may have larger total spin than lanthanide and actinide atoms. In both cases we propose to measure the spectrum of low-lying states (EPR or NMR measurements) and also to measure magnetization and magnetic susceptibility at low temperatures (about 1-2 K). Though experimental difficulties may arise on the way to realization of these experiments, we believe that the expected physical phenomena are worthwhile to study.

The third class is magnetic dots used in experiments on magnetic tunneling $[24,25]$.

Experimenters should choose optimal values of $J$ to ensure the validity of the quasiclassical approach: reliable separation of the $N$-fold multiplets and simultaneously not too small values of the tunneling exponent $\exp (-c J)$ with $c$ $\leqslant 0.55$ for the cubic symmetry and $c \leqslant 0.29$ for the icosahedral symmetry.

An interesting experimental and maybe technical application of our system is the excitation of magnetic oscillations in a narrow spectral region by pulses of external magnetic field. The frequency of these oscillations ranges from $10^{7}$ to $10^{11} \mathrm{~Hz}$.

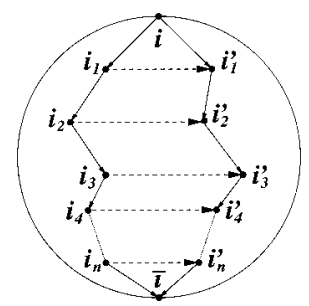

FIG. 21. Transformation of a tunneling path $i \rightarrow i_{1} \rightarrow \cdots \rightarrow i_{n}$ $\rightarrow \bar{l}$ onto $i \rightarrow i_{1}^{\prime} \rightarrow \cdots \rightarrow i_{n}^{\prime} \rightarrow \bar{l}$. A solid angle of the filled area is $4 \pi q / p$.

\section{ACKNOWLEDGMENTS}

We are thankful to E. Müller-Hartmann and G. S. Uhrig who initiated this subject, and to V. V. Dobrovitski for useful discussions. V.K. is grateful to I. V. Lavrinenko for the discussions on group theory and to J. D. Louck for the valuable references on earlier works and discussion on this subject. This work was supported by the NSF under Grant No. DMR97-05182 and by the U.S. DOE under Grant No. DE-F0396ER45598.

\section{APPENDIX A: CHARACTERS OF THE MAIN REPRESENTATION}

The character of the identity transformation $E$ is trivial, $\chi(E)=\operatorname{dim}(W)=N$. For a half integer $J$, one has to find projective representations of the corresponding group with the factor set of $\{ \pm 1\}$ or, equivalently, linear representations of the group extended by a group $\{E, Q \equiv \exp (i 2 \pi J) E\}$ (the so-called two-valued representations), where $Q$ is a rotation through an angle of $2 \pi$ (here we adopt the notations of Ref. [5]). Obviously $\chi(Q)=\cos (2 \pi J) \operatorname{dim}(W)=\cos (2 \pi J) N$. Other elements having nonzero characters are the rotations with respect to the axes passing through the directions belonging to the set $\mathcal{C}(G, p)$. Actually, it is sufficient to consider only one element from each class of conjugate elements. To calculate the corresponding characters we employ the following trick.

Let us consider an element of $P$ which, in our basis, corresponds to a rotation $C_{p}^{q}$ with respect to a $p$-fold axis. A set of rotations with respect to this axis forms a cyclic subgroup of $P$, and $q$ is a power of the generator of the subgroup $C_{p}$ (minimal nontrivial rotation); $q$ may take any integer value. For a given configuration $\mathcal{C}(G, p)$ a nonzero character may occur only if $C_{p}^{q}$ leaves at least two of the extrema $i$ and $\bar{l}$ unmoved. It means that either the rotation axis passes through $i$ and $\bar{l}$, and $q \neq p n$ or the rotation is trivial: $q$ $=p n, n$ is an integer. Let us choose a tunneling path connecting $i$ and $\bar{l}$, and passing through intermediate nearestneighbor extrema $i \rightarrow i_{1} \rightarrow \cdots \rightarrow i_{n} \rightarrow \bar{l}$ (see Fig. 21). Note that some of the minima may coincide, that is, $i_{j}=i_{k}$ for some pairs $j \neq k$. The rotation $C_{p}^{q}$ transfers each extremum $i_{j}(j=1,2, \ldots, n)$ into $i_{j}^{\prime}$ leaving $i$ and $\bar{l}$ unchanged. The two paths form a closed loop on the sphere which subtends the solid angle of $4 \pi q / p$. This fact leads to a relation for the oriented sum of the phases along the circuit: 


$$
\begin{aligned}
& \sum_{j=1}^{n-1}\left(\phi_{i_{j}, i_{j+1}}-\phi_{i_{j}^{\prime}, i_{j+1}^{\prime}}\right)+\phi_{i, i_{1}}-\phi_{i, i_{1}^{\prime}}+\phi_{i_{n}, l}-\phi_{i_{n}^{\prime}, \bar{l}} \\
& \quad=J \frac{4 \pi q}{p} .
\end{aligned}
$$

The same rotation $C_{p}^{q}$ transforms the phases in the following way: $\phi_{i_{j}, i_{j+1}} \mapsto \phi_{i_{j}^{\prime}, i_{j+1}^{\prime}}, j=1,2, \ldots, n-1, \phi_{i, i_{1}} \mapsto \phi_{i, i_{1}^{\prime}}$, $\phi_{i_{n}, l} \mapsto \phi_{i_{n}^{\prime},-}$. To keep the phases unchanged (modulo $2 \pi$ ) and the Hamiltonian invariant one should apply a gauge transformation $\mathcal{U}=\operatorname{diag}\left\{\exp \left(i \alpha_{1}\right), \ldots, \exp \left(\alpha_{N}\right)\right\}$, then the mappings turn into equations:

$$
\begin{gathered}
\phi_{i_{j}, i_{j+1}}=\phi_{i_{j}^{\prime}, i_{j+1}^{\prime}}+\alpha_{i_{j}}-\alpha_{i_{j+1}}, \\
\phi_{i, i_{1}}=\phi_{i, i_{1}^{\prime}}+\alpha_{i}-\alpha_{i_{1}}, \\
\phi_{i_{n},-l}=\phi_{i_{n}^{\prime},-}+\alpha_{i_{n}}-\alpha_{l}^{-} .
\end{gathered}
$$

Substituting these equations into Eq. (A1) gives

$$
\alpha_{i}-\alpha_{l}^{-}=J \frac{4 \pi q}{p},
$$

which leads to

$$
\alpha_{i_{0}}=J \frac{2 \pi q}{p}+\delta, \alpha_{\iota_{0}}^{-}=-J \frac{2 \pi q}{p}+\delta
$$

Multiplying $\mathcal{U}$ with $\exp (-i \delta)$ removes the common factor $\delta$ in Eq. (A3). Thus, the transformation $\mathcal{W}=\exp (-i \delta) \mathcal{U} C_{p}^{q}$ of the configuration space has only two diagonal elements $\{\mathcal{W}\}_{i i}=\{\mathcal{W}\} \frac{*}{l l}=\exp (i 2 \pi J q / p)$. The character of the element $\mathcal{W}$ is $2 \cos (J 2 \pi q / p)$. All other characters are zeros since the corresponding rotations do not leave any state $|k\rangle$ of the configuration invariant. The characters of the representation do not depend upon the gauge: $\operatorname{Tr}\left(\mathcal{W}^{\prime}\right)=\operatorname{Tr}\left(\mathcal{U} \mathcal{W U}^{\dagger}\right)$ $=\operatorname{Tr}(\mathcal{W})$. Thus, one can study the reduction of the representation $W$ in any specific gauge without loss of generality.

\section{APPENDIX B: AN EXAMPLE OF A HAMILTONIAN FOR THE $\mathcal{C}(0,4)$ CONFIGURATION AND CALCULATION OF ITS SPECTRA}

In this appendix we consider a detailed construction of the reduced Hamiltonian for the $\mathcal{C}(\mathbf{O}, 4)$ configuration. First we define the connectivity matrix of the system determining which minima ought to be connected by tunneling paths. This is usually done by connecting the nearest-neighbor minima. In some cases, however, the geometric closeness on the sphere is not a good criterion for connecting minima. A "surefire" criterion is the path integral approach which determines the amplitude of a spin transition from one localized state to another by summation of the contributions of all trajectories connecting the minima. This technique gives the exact solution of the problem, but it is very complicated. We could use instead a semiclassical method of finding trajectories with minimal imaginary classical action, but the action is not known itself. However, the symmetry of the system is of great help and is used throughout the paper to assess the connectivity structure. An example in which the geometrically next-nearest neighbors must be incorporated into the connectivity matrix together with the nearest neighbors is discussed in Sec. V B.

The connectivity matrix of the $\mathcal{C}(\mathbf{O}, 4)$ configuration is quite simple since only the geometric nearest neighbors should be connected. For the minima enumeration given in Fig. 1 the connectivity matrix is

$$
\left|\begin{array}{lll}
\hat{\mathbf{0}} & \hat{\mathbf{1}} & \hat{\mathbf{1}} \\
\hat{\mathbf{1}} & \hat{\mathbf{0}} & \hat{\mathbf{1}} \\
\hat{\mathbf{1}} & \hat{\mathbf{1}} & \hat{\mathbf{0}}
\end{array}\right|, \quad \hat{\mathbf{0}}=\left|\begin{array}{ll}
0 & 0 \\
0 & 0
\end{array}\right|, \quad \hat{\mathbf{1}}=\left|\begin{array}{ll}
1 & 1 \\
1 & 1
\end{array}\right| .
$$

This is, actually, the Hamiltonian for $J=0(\bmod 4)$, without a multiplier of $w$, since the phase structure is absent or of no importance for the respective cases. For all other $J$ 's one should find the 12 phases of the tunneling amplitudes. Five of the phases may be set to zero due to the gauge freedom with the only constraint that Eq. (12) must be satisfied. In our sample case, we null the following ones: $\phi_{1,3}, \phi_{1,4}$, $\phi_{1,5}, \phi_{1,6}, \phi_{2,5}$. The rest of the phases are obtained from the seven independent plaquettes [each plaquette gives an equation of the type of the Eq. (12)]; these are $\phi_{2,3}=-\pi J, \phi_{2,4}$ $=\pi J, \phi_{2,6}=2 \pi J, \phi_{3,5}=\pi J / 2, \phi_{3,6}=-\pi J / 2, \phi_{4,5}=-\pi J / 2$, $\phi_{4,6}=\pi J / 2$. Thus, the defined Hamiltonian is

$$
\mathcal{H}_{4}^{\mathbf{O}}=\left|\begin{array}{cccccc}
0 & 0 & 1 & 1 & 1 & 1 \\
0 & 0 & e^{-i \pi J} & e^{i \pi J} & 1 & e^{i 2 \pi J} \\
1 & e^{i \pi J} & 0 & 0 & e^{i \pi J / 2} & e^{-i \pi J / 2} \\
1 & e^{-i \pi J} & 0 & 0 & e^{-i \pi J / 2} & e^{i \pi J / 2} \\
1 & 1 & e^{-i \pi J / 2} & e^{i \pi J / 2} & 0 & 0 \\
1 & e^{-i 2 \pi J} & e^{i \pi J / 2} & e^{-i \pi J / 2} & 0 & 0
\end{array}\right| .
$$

Finally, one needs to find the eigenvalues of the Hamiltonian. The diagonalization can be performed by any symbolic solving system or even "manually" since the Hamiltonian can be factorized. Also, a trick of a purely geometric origin can be used: if one views Hamiltonian $\mathcal{H}[w=1$ and $\left|(\mathcal{H})_{i j}\right|=1$ if $\left.(\mathcal{H})_{i j} \neq 0\right]$ as a weighted connectivity matrix of a graph, then

$$
\sum_{i} g_{i} E_{i}^{n}=\operatorname{Tr} \mathcal{H}^{n}=2 \sum_{j} \sum_{k_{j}} \cos \left(\Omega_{k_{j}}\right) \equiv I_{n},
$$

where $n=0,1,2, \ldots, E_{i}$ is the $i$ th distinct eigenvalue of $\mathcal{H}$ of multiplicity $g_{i}$, the first sum on the right-hand side is over the vertices of the graph, the second is over all closed loops of $n$ walks running through vertex $j$, and $\Omega_{k_{j}}$ is the flux passing through the $k_{j}$ th loop. If $(\mathcal{H})_{i i} \neq 0, i=1, \ldots, N$, some extra weights need to be applied for each loop. An advantage of formula (B3) is that $I_{n}$ are gauge invariant since all $\Omega_{k_{j}}$ are gauge invariant. Applied to Hamiltonian (B2) for $n<4$, the formula yields

$$
\sum_{i} g_{i}=6, \sum_{i} g_{i} E_{i}=0,
$$




$$
\sum_{i} g_{i} E_{i}^{2}=24, \sum_{i} g_{i} E_{i}^{3}=48 \cos (\pi J / 2)
$$

Let us find the spectrum of Hamiltonian (B2) for $J=0$. We know from Sec. IV B 1 that $g_{1}=1, g_{2}=2$, and $g_{3}=3$. Too, it is clear that $E_{1}=4$ [sum of the matrix elements in each row or column of Hamiltonian (B2) is equal to 4 for $J=0]$. Two equations for $E_{2}$ and $E_{3}$ are

$$
4+2 E_{2}+3 E_{3}=0,16+2 E_{2}^{2}+3 E_{3}^{2}=24 .
$$

Solving Eq. (B5) and checking the roots against the last equation of Eq. (B4) gives $E_{2}=-2$ and $E_{3}=0$. For $J=2$, the spectrum is inverted (see Sec. V): $E_{1}=-4, E_{2}=0$, and $E_{3}=2$. For all other $J$ 's the solution is trivial since there are only two distinct eigenvalues.

\section{APPENDIX C: \\ LOW-TEMPERATURE MAGNETIC SUSCEPTIBILITY}

For high temperatures $k_{B} T>w$, the moments are purely classical and show Curie magnetic susceptibility:

$$
\chi_{C}=\left(\mu_{B} g J\right)^{2} /\left(d k_{B} T\right),
$$

where $d$ is the dimensionality of the system, $\mu_{B}$ is the Bohr magneton, $k_{B}$ is the Boltzmann constant, and $g$ is the gyromagnetic ratio. For low temperatures $k_{B} T<w$, the quantum effects change the response drastically. The susceptibility saturates for the classes without magnetic moment in the ground state to the value

$$
\chi_{s}=\frac{1}{g_{0}} \sum_{i=1}^{g_{0}} \chi_{i},
$$

where $g_{0}$ is the degeneracy of the ground state and $\chi_{i}$ is the susceptibility of the $i$ th member of the ground-state multiplet. For the classes with the magnetic moment in the ground state Curie susceptibility persists at $k_{B} T<w$, but its slope is different:

$$
\chi_{l}=\frac{1}{g_{0}} \sum_{i=1}^{g_{0}} m_{i}^{2} /\left(k_{B} T\right)
$$

where $m_{i}$ is the moment of the $i$ th member of the groundstate multiplet.

\section{APPENDIX D: ON THE INTERSECTION OF THE MULTIPLETS OF $\mathcal{C}(0,4)$ AND $\mathcal{C}(0,3)$}

In the close vicinity of a minimum of $\mathcal{C}(\mathbf{O}, 4)$ and $\mathcal{C}(\mathbf{O}, 3)$ configurations, Hamiltonian (6) has the following form:
TABLE XVI. Spectra of a "hybrid" $\mathcal{C}(\mathbf{O}, 4)+\mathcal{C}(\mathbf{O}, 3)$ configuration.

\begin{tabular}{lc}
\hline \hline$J$ & Eigenvalues (degeneracies) \\
\hline 0 & $\pm 2 \sqrt{3} w(1,1), \pm 2 w(3,3), 0(6)$ \\
1 & $\pm(1+\sqrt{3}) w(3,3), \pm(1-\sqrt{3}) w(3,3), 0(2)$ \\
2 & $\pm \sqrt{6} w(2,2), \pm 2 w(3,3), 0(4)$ \\
3 & $\pm 2 w(6,6), 0(2)$ \\
$1 / 2$ & $\pm w \sqrt{6+2 \sqrt{3}}(2,2), \pm w \sqrt{3-\sqrt{3}}(4,4), 0(2)$ \\
$3 / 2$ & $\pm w \sqrt{6}(4,4), 0(6)$ \\
$5 / 2$ & $\pm w \sqrt{6-2 \sqrt{3}}(2,2), \pm w \sqrt{3+\sqrt{3}}(4,4), 0(2)$ \\
\hline \hline
\end{tabular}

$$
\mathcal{H}_{2}^{\mathbf{0} 4}=-a-b+(2 a+3 b)\left(x^{2}+y^{2}\right),
$$

$$
\mathcal{H}_{2}^{\mathbf{O} 3}=-\frac{1}{3} a-\frac{11}{9} b+\frac{4}{3}(4 b-a)\left(x^{2}+y^{2}\right),
$$

where $x$ and $y$ are local Cartesian coordinates. Treating these terms as the effective potential energy of the quantummechanical problem, one can identify it with that for a twodimensional isotropic harmonic oscillator [the kinetic energy is due to the Wess-Zumino term [29] or Berry phase, its exact form is of no importance here; it suffices to know that this term is identical for both Eqs. (D1) and (D2), thus providing identical effective masses $M]$. The potential energies of the two harmonic oscillator problems are equal on a line $b=3 a$. The squares of the effective frequencies are $22 a / M$ and $88 a /(3 M)(a>0)$, respectively, for $\mathcal{C}(\mathbf{O}, 4)$ and $\mathcal{C}(\mathbf{O}, 3)$, on this line. Hence, the energy of the ground as well as the spacing between successive levels is larger for the $\mathcal{C}(\mathbf{O}, 3)$ configuration on this line. These arguments qualitatively explain the deviation of the line $b=3 a$ from the point of the level crossing towards the sixfold coordination region.

The boundary of the transition from one configuration to another (a line in our two-dimensional $a-b$ space) marks a singularity in the "flow" of the level multiplicities across the parameter space. The levels of two different coordinations must match exactly at this surface. We find the spectra of this intermediate configuration in the case of $N=N(\mathbf{O}, 4)$ $+N(\mathbf{O}, 3)=14$.

The surface of the sphere is covered with 12 congruent even-sided plaquettes. Hence, the period of spectra is $J=6$ and all spectra are symmetric (see Sec. V). The spectra of the 14-fold configuration are collected in Table XVI for the nonequivalent $J$ 's.
[1] F.D.M. Haldane, Phys. Rev. Lett. 50, 1153 (1983); Phys. Lett. 93A, 464 (1983); Bull. Am. Phys. Soc. 27, 181 (1982).

[2] W.G. Harter and C.W. Patterson, J. Chem. Phys. 66, 4872 (1977); C.W. Patterson and W.G. Harter, ibid. 66, 4886 (1977).
[3] We are indebted to B. I. Zhilinskii, who directed us to these works.

[4] D.J. Newman, Adv. Phys. 20, 197 (1971).

[5] L.D. Landau and E.M. Lifshits, Quantum Mechanics - Nonrelativistic Theory (Pergamon Press, London, 1987). 
[6] H.-B. Braun and D. Loss, Phys. Rev. B 53, 3237 (1996).

[7] V.A. Kalatsky, E. Müller-Hartmann, V.L. Pokrovsky, and G.S. Uhrig, Phys. Rev. Lett. 80, 1304 (1998).

[8] M.V. Berry, Proc. R. Soc. London, Ser. A 392, 45 (1984).

[9] P.G. Harper, Proc. Phys. Soc. London, Sect. A 68, 874 (1955).

[10] M.Ya. Azbel', Zh. Éksp. Teor. Fiz. 46, 939 (1964) [Sov. Phys. JETP 19, 634 (1964)]; R.D. Hofstadter, Phys. Rev. B 14, 2239 (1976); D.J. Thouless, ibid. 28, 4272 (1983); P.B. Wiegmann and A.V. Zabrodin, Phys. Rev. Lett. 72, 1890 (1994).

[11] J.J. Sakurai, Modern Quantum Mechanics (Addison-Wesley, Reading, MA, 1994), Chap. 4.4.

[12] For an introduction to cohomology of groups and extensions one can refer to J.A. de Azcárraga and J.M. Izquierdo, Lie groups, Lie algebras, Cohomology and Some Applications in Physics (Cambridge University Press, New York, 1995).

[13] A.A. Kirillov, Elements of the Theory of Representations (Springer-Verlag, New York, 1976), Part 2.

[14] D. Loss, D.P. DiVincenzo, and G. Grinstein, Phys. Rev. Lett. 69, 3232 (1992).

[15] V.A. Kalatsky and V.L. Pokrovsky, Europhys. Lett. 44, 539 (1998).

[16] H. Friedrich and D. Wintgen, Phys. Rep. 183, 37 (1989).

[17] M.T. Hutchings, in Solid State Physics: Advances in Research and Applications, edited by F. Seitz and D. Turnbull (Academic, New York, 1964), Vol. 16, p. 227.
[18] K.R. Lea, M.J.M. Leask, and W.P. Wolf, J. Phys. Chem. Solids 23, 1381 (1962).

[19] K. Fox, H.W. Galbraith, B.J. Krohn, and J.D. Louck, Phys. Rev. A 15, 1363 (1977).

[20] K.W.H. Stevens, Proc. Phys. Soc. London, Sect. A 65, 209 (1952).

[21] B.K. Cho, B.N. Harmon, D.C. Johnston, and P.C. Canfield, Phys. Rev. B 53, 2217 (1996).

[22] J.M. Van Ruitenbeek, D.A. Van Leeuwen, and L.J. De Jongh, in Physics and Chemistry of Metal Cluster Compounds, edited by L.J. De Jongh (Kluwer Academic Publishers, Dordrecht, 1994), Vol. 18, p. 227.

[23] D.C. Douglass, J.P. Bucher, and L.A. Bloomfield, Phys. Rev. Lett. 68, 1774 (1992).

[24] P.C.E. Stamp, E.M. Chudnovsky, and B. Barbara, Int. J. Mod. Phys. B 6, 1355 (1992).

[25] J.I. Martín, M. Vélez, J. Nogués, and Ivan K. Schuller, Phys. Rev. Lett. 79, 1929 (1997).

[26] D.G. Naugle (private communication).

[27] We are indebted to V.V. Dobrovitski, who indicated the importance of this effect.

[28] J.P. Aldridge, H. Filip, H. Flicker, R.F. Holland, R.S. McDowell, and N.G. Nerson, J. Mol. Spectrosc. 58, 167 (1975).

[29] E. Fradkin, Field Theories in Condensed Matter (AddisonWesley, Reading, MA, 1991), Chap. 5. 